\title{
Thick-Walled Cylinder Theory Applied on a Conical Wedge Anchorage
}

\author{
Anders Bennitz ${ }^{1}$, Niklas Grip ${ }^{2 *}$ and Jacob W. Schmidt ${ }^{3}$ \\ ${ }^{1}$ Division of Structural Engineering, Department of Civil, Mining and Environmental Engineering, \\ Luleå University of Technology, SE-971 87 Luleå, Sweden, Anders.Bennitz@ltu.se. \\ ${ }^{2}$ Department of Mathematics, Luleå University of Technology, SE-971 87 Luleå, Sweden, Niklas.Grip@ltu.se. \\ ${ }^{3}$ Department of Civil Engineering, Technical University of Denmark, DK-2800 Kgs. Lyngby, Denmark, jws@byg.dtu.dk.
}

July 17, 2010

\begin{abstract}
Conical wedge anchorages are frequently used to anchor steel tendons in prestressing applications within the construction industry. To replace the steel tendons with noncorrosive and low weight FRPs (Fiber Reinforced Polymers), the different mechanical interactions between the steel and FRPs call for further development of the anchorage.

In this paper, we derive and examine an analytical model for the internal stresses and strains within the anchorage for a prescribed presetting distance. This model is derived from the theory of thick walled cylinders under the assumptions regarding plane stress and axial symmetry. We simplify the resulting system of ten nonlinear equations and derive a method for solving them numerically. A comparison of plotted results for three different angles on the wedge's outer surface and six different presetting distances follows.

These results are also compared to both axi-symmetric and 3D FE (Finite Element) models. Analytical and FE axi-symmetric models show good correspondence, though compared to the 3D FE model, they show a clear difference in the predicted radial stress distribution on the FRP. Thus, the derived analytical model can be a useful and faster alternative to FE modeling of axi-symmetric anchorages. However, the model is of more restricted value and should be complemented by, for example, 3D FE models for other designs.
\end{abstract}

Keywords: Wedge, Anchorage, Thick-walled cylinder, FE-Finite Element, Bisection method, Prestress, FRP, Concrete

${ }^{*}$ The author was partially supported by the Swedish Research Council (project registration number 2004-3862). 


\section{Background}

Concrete is a strong material as long as it is loaded in compression, but it can only carry onetenth of the same load in tension. Since the material is often used to span large distances and support traffic loads (bridges, parking garages) or people and furniture in multi-story buildings, it is also subjected to high tensile forces in the lower part of the beam/slab. This tension is the reason why steel reinforcement is cast into the structure. To further increase capacity the reinforcement can be tensioned before the concrete is cast and then released once the concrete has hardened, thus also applying a compressive stress in the lower part of the beam/slab. With this prestressing force applied, some of the load must be applied to return the lower part to a state of zero stress; hence, the capacity of the structure concerning cracking and deflection has been increased. Some of the most important literature written in the area of prestressing is CM91, LB82. Today, an exchange of the steel strands used in prestressing applications is being requested. Steel is generally highly corrosive and its weight makes it heavy to work with. An excellent alternative that has emerged in the last 10-20 years is FRPs (Fibre Reinforced Polymers), of which CFRP (Carbon FRP) is the most suitable concerning mechanical properties. CFRP is strong, stiff, resistant to environmental exposure and lightweight. However, some development is necessary to industrialize this new application of the CFRP. Finding a suitable anchorage that can grip the CFRP rod and handle the high tensile forces involved in the prestressing process is critical. Unlike steel, FRPs are elastic until they rupture as well as being weaker in the transversal direction than in the longitudinal. The first difference causes a disability for the FRPs to redistribute stress concentrations caused by the internally threaded conical wedges, traditionally used to anchor steel strands. Instead of the threads, all stresses must be transferred by friction between two relatively smooth surfaces if a CFRP strand is to be anchored. The second difference, the low transverse compressive capacity of the FRPs, makes gripping even more difficult, since it does not allow an increase of the radial gripping force above a certain value.

To date, research in the area has only been reported by two Canadian research groups. One that in SAS98 introduced a difference in angle between the conical outer faces of the wedges and the inner conical face of the barrel. This difference causes the anchorage to first grip around the CFRP-strand in the back of the anchorage, thus avoiding high principal stresses in the front of the anchorage where the tensile stresses in the rod are greatest. A version of the developed anchorage is then modeled and tested by the other research group in AMSP01a, AMSP01b. Later, this group also developed a new anchorage with a curved conical face, again to shift initial transfer of stresses towards the back of the anchorage AMSP06, AMSP07.

The work presented in this paper is inspired by these publications and the models used therein. Emphasis is put on presenting a well-founded and verified solution to a problem closely related to the analytical model suggested in AMSP01a, designed for computing the variation of radial pressure along the length of the anchorage. We also compare the results from differences in angles between the barrel and wedges as well as a comparison with the corresponding results for both an axi-symmetric and a 3D (Three-Dimensional) FE (Finite Element) model of the anchorage. As assistance in the evaluation some of the results and findings are put side by side with results from AMSP01a, AMSP07 for further verification, or as a source for discussion.

\section{Research Identification}

For the reasons explained in last section, we have developed a conical wedge anchorage for circular CFRP rods. These anchorages consist of an outer barrel in steel and three wedges in aluminium. As tension is applied to the rod, the wedges are pulled into the barrel and grip harder around the rod, thus increasing the capacity to transfer the load by friction. In this 


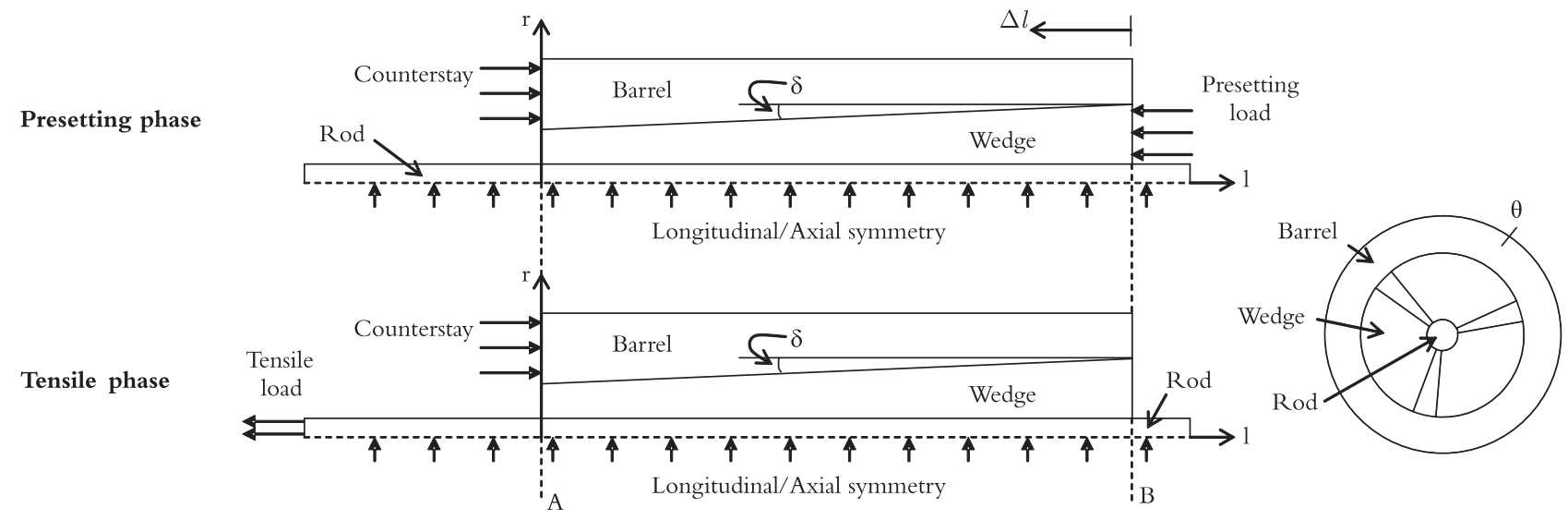

Figure 1: Cross-sections of anchorage during presetting and tensile loading phases.

research the presetting phase is further investigated, i.e. when the wedges are pushed slightly into the barrel from behind to ensure that an initial grip exists when the tensile force is applied to the rod. Figure 1 shows the setup in these two loading phases. Of note are the radial stresses acting on the rod (denoted $P_{1}$ from now on, see Figure 2). Since the longitudinal forces on the rod decrease with the distance from the front of the anchorage, the barrel and wedge should be designed to partially compensate for this with a radial stress $P_{1}$ that increases from the front to the back of the anchorage so that the total load is roughly constant.

Hence, how well an analytical model can describe the variation of radial stress onto the rod along the length of the anchorage is of interest. If such a model is found reliable, it can then be used to reshape and further develop the design of the anchorage. The analytical and finite element models compared in this paper rely on the geometric and material properties listed in Table 1. with geometrical notations from Figure 1. The subscripts o and i denote outer and inner radii, and the subscripts $\mathrm{A}$ and $\mathrm{B}$ note the front and back ends of the anchorage. $E$ is the modulus of elasticity for each material and $\nu$ is the Poisson ratio.

\section{Theory}

Because parts of the anchorage are thick compared to their radii, the thin-walled cylinder theory provides an insufficient description of the distribution of stresses. Instead, we propose a model based on the thick-walled cylinder theory, where for a prescribed presetting distance $\Delta l$, the radial stresses and strains on each surface are calculated for each radial cross- section along the length of the anchorage.

The thick-walled cylinder theory is a 3D theory capable of producing closed analytical solutions due to the limitations applied. Several publications on the subject of solid mechanics include a derivation of the theory's basic equations [BC95, Lun00. Wan53 provided the

Table 1: Geometrical and material properties

\begin{tabular}{|c|c|c|c|c|c|c|c|c|c|c|c|}
\hline Part & $l[\mathrm{~mm}]$ & $r_{\mathrm{oA}}[\mathrm{mm}]$ & $r_{\mathrm{iA}}[\mathrm{mm}]$ & $r_{\mathrm{oB}}[\mathrm{mm}]$ & $r_{\mathrm{iB}}[\mathrm{mm}]$ & $\delta\left[{ }^{\circ}\right]$ & $\theta\left[{ }^{\circ}\right]$ & Material & $\rho\left[\mathrm{kg} / \mathrm{m}^{3}\right]$ & $E[\mathrm{GPa}]$ & $\nu[-]$ \\
\hline Barrel & 100 & 24.7 & 7.26 & 24.7 & 12.5 & 3 & 360 & Steel & 7800 & 210 & 0.3 \\
\hline Wedge & 100 & $7.26,7.08,6.91 \mathrm{a}$ & 4 & 12.5 & 4 & $3,3.1,3.2$ & $360 / 110^{b}$ & Aluminium & 2700 & 70 & 0.34 \\
\hline Rod & 150 & 4 & - & 4 & - & - & 360 & CFRP & 1610 & $10 / 16 \sqrt[5]{0}$ & $0 . \sqrt{d}$ \\
\hline
\end{tabular}

\footnotetext{
${ }^{a}$ Three different angles on the outer surface of the wedges are used, which results in three different outer radii of the wedge in point A.

${ }^{b}$ In the analytical and axi-symmetric FE-model one wedge cover $360^{\circ}$ while three wedges cover $110^{\circ}$ each in the $3 \mathrm{D}$ FE-model.

${ }^{c}$ In the FE-models the CFRP has the higher value on $\mathrm{E}$ in the longitudinal direction and the lower in the radial direction.

${ }^{d}$ We list $\nu$ only for the radial and circumferential stresses present in our analytical model.
} 
inspiration in this paper to produce the set of equations solved. Also, AMSP07, SS06 have used the theory in different shapes to evaluate the behaviour of the anchorages.

\subsection{Simplifications and limitations}

Several important simplifications are built into the theory of thick-walled cylinders. These will certainly influence the results and should be considered when interpreting the results.

\subsubsection{Axial symmetry}

Axial symmetry means that the medium modeled is consistent throughout the $360^{\circ}$ describing the circumference of a symmetry axis. For the CFRP rod and the barrel, this axis of symmetry is positioned along the core of the rod. The anchorage is not axially symmetric, however, because of the air gaps separating the three wedges (see Figure 1).

In assuming axial symmetry for the entire model, it is accordingly assumed that no air gap exists and that one wedge spans the entire circumference. Hence, the wedge will handle forces as an arch and the radial stresses onto the rod will decrease compared to a 3D case.

For calculation purposes, the consequences in assuming axial symmetry are that the forces acting on faces normal to the circumferential direction are constant, or with the standard notation explained in Figure 10 (a) of Appendix A, $\sigma_{\theta}, \tau_{\theta r}$ and $\tau_{\theta l}$ depend only on the longitudinal position $l$ in Figure 2. Similarly, all radial and circumferential displacements are constant along the circumferential axis, but vary with $l$ and in the radial direction (see Appendix $\mathrm{A}$, Figure $10(\mathrm{~b}))$.

\subsubsection{Plane stress}

This paper assumes the use of plane stress, i.e. no longitudinal stresses are present in the model and $\sigma_{1}=0$ (more extensively described in Appendix A, Figure 11). Consequently, the anchorage is more or less described as a 2D (Two-Dimensional) model. The dimensions used in the calculations are the radial and circumferential directions. A consequence is that one calculation must be done at each point along the longitudinal axis where a result is sought.

These results are also independent from any stresses resulting from neighbouring sections; thus, the materials are free to swell in the longitudinal direction without restrictions.

In mathematical terms, the simplification means that all shear forces acting in the longitudinal direction are equal to zero, i.e. $\tau_{\mathrm{rl}}=\tau_{\theta \mathrm{l}}=0$; hence, $\tau_{\mathrm{lr}}=\tau_{\mathrm{l} \theta}=0$. Further, no compatibility equation in the longitudinal direction is necessary, since the strain will be constant in the longitudinal direction for a point in the $r-\theta$ plane.

\subsubsection{Elastic and isotropic materials}

Metallic materials generally have a plastic behaviour, where the material after a certain amount of strain deforms more rapidly without increasing the capability of resisting stresses, but merely keeps it constant. By assuming elastic materials no such limit exists, i.e. the material will experience a linearly increasing stress with increasing strain and the parameter governing this relation is the modulus of elasticity, $E$.

This assumption will keep the deformations relatively small without the material having any limit concerning what stress it can handle.

In reality, the metals are isotropic and perfectly described by this assumption. CFRP, on the other hand, is strongly orthotropic with a stiff behaviour in the longitudinal direction, along the fibres, and softer in the radial direction. This orthotropicity is used in the FE models described in Section 5 and 6. whereas the analytical model described in Section 4 does not 
allow for orthotropic modeling of the materials, since it only handles radial and circumferential stresses, so there only the lower (transverse) $E$-value of Table 1 is needed.

\section{Analytical results assuming axial symmetry}

Assuming axial symmetry, we want to compute the new surface radii $n_{k}$ and the pressures $P_{k}$ at longitudinal position $l$, as depicted in Figure 2. At this longitudinal position, the wedge is compressed from inner and outer radii $r_{\text {wi }}$ and $r_{\text {wo }}$ to the new radii $n_{1}$ and $n_{2}$. Similarly, the original barrel inner and outer radii $r_{\mathrm{bi}}$ and $r_{\mathrm{bo}}$ at this cross-section have changed to $n_{2}$ (for $r_{\mathrm{bi}}$ ) and $n_{3}$ (for $r_{\mathrm{bo}}$ ). For the rod, finally, the original radius $r_{\text {ro }}$ is compressed to the new radius $n_{1}$. Note that with this terminology, everywhere where the wedge's outer surface and the barrel's inner surface touch, $r_{\text {wo }} \geq r_{\text {bi }}$ and $r_{\text {wi }}=r_{\text {ro }}$.

Appendix A shows how a plane stress scenario and the application of Hooke's generalized law to an infinitesimal 3D-element gives the following relationship between these radii and pressures, with the notations $E_{\mathrm{r}}$ and $\nu_{\mathrm{r}}$ for Young's modulus and the Poisson ratio of the rod, $E_{\mathrm{w}}$ and $\nu_{\mathrm{w}}$ for the wedge and $E_{\mathrm{b}}$ and $\nu_{\mathrm{b}}$ for the barrel:

$$
\begin{array}{ll}
r_{\mathrm{ro}}-n_{1}=P_{1} n_{1} \frac{1-\nu_{\mathrm{r}}}{E_{\mathrm{r}}} & P_{1}, n_{1} \\
r_{\mathrm{wi}}-n_{1}=\frac{n_{1}}{E_{\mathrm{w}}\left(n_{2}^{2}-n_{1}^{2}\right)}\left(2 P_{2} n_{2}^{2}-P_{1}\left(\left(1-\nu_{\mathrm{w}}\right) n_{1}^{2}+\left(1+\nu_{\mathrm{w}}\right) n_{2}^{2}\right)\right) & P_{1}, P_{2}, n_{1}, n_{2} \\
r_{\mathrm{wo}}-n_{2}=\frac{n_{2}}{E_{\mathrm{w}}\left(n_{2}^{2}-n_{1}^{2}\right)}\left(P_{2}\left(\left(1-\nu_{\mathrm{w}}\right) n_{2}^{2}+\left(1+\nu_{\mathrm{w}}\right) n_{1}^{2}\right)-2 P_{1} n_{1}^{2}\right) & P_{1}, P_{2}, n_{1}, n_{2} \\
r_{\mathrm{bi}}-n_{2}=-\frac{P_{2} n_{2}}{E_{\mathrm{b}}\left(n_{3}^{2}-n_{2}^{2}\right)}\left(\left(1-\nu_{\mathrm{b}}\right) n_{2}^{2}+\left(1+\nu_{\mathrm{b}}\right) n_{3}^{2}\right) & P_{2}, n_{2}, n_{3} \\
r_{\mathrm{bo}}-n_{3}=-\frac{2 P_{2} n_{2}^{2} n_{3}}{E_{\mathrm{b}}\left(n_{3}^{2}-n_{2}^{2}\right)} & P_{2}, n_{2}, n_{3}
\end{array}
$$

We solve equations (11) in the following five steps:

1. From equation (1a) we get

$$
n_{1}\left(P_{1}\right)=\frac{E_{\mathrm{r}} r_{\mathrm{ro}}}{E_{\mathrm{r}}+P_{1}\left(1-\nu_{\mathrm{r}}\right)} .
$$

2. From (1b) and the fact that $n_{2}>0$, we get

$$
n_{2}\left(P_{1}, P_{2}\right)=\sqrt{\frac{E_{\mathrm{w}} r_{\mathrm{wi}}-\left(E_{\mathrm{w}}+P_{1}\left(1-\nu_{\mathrm{w}}\right)\right) n_{1}\left(P_{1}\right)}{E_{\mathrm{w}} r_{\mathrm{wi}}+\left(P_{1}\left(1+\nu_{\mathrm{w}}\right)-2 P_{2}-E_{\mathrm{w}}\right) n_{1}\left(P_{1}\right)}} n_{1}\left(P_{1}\right) .
$$

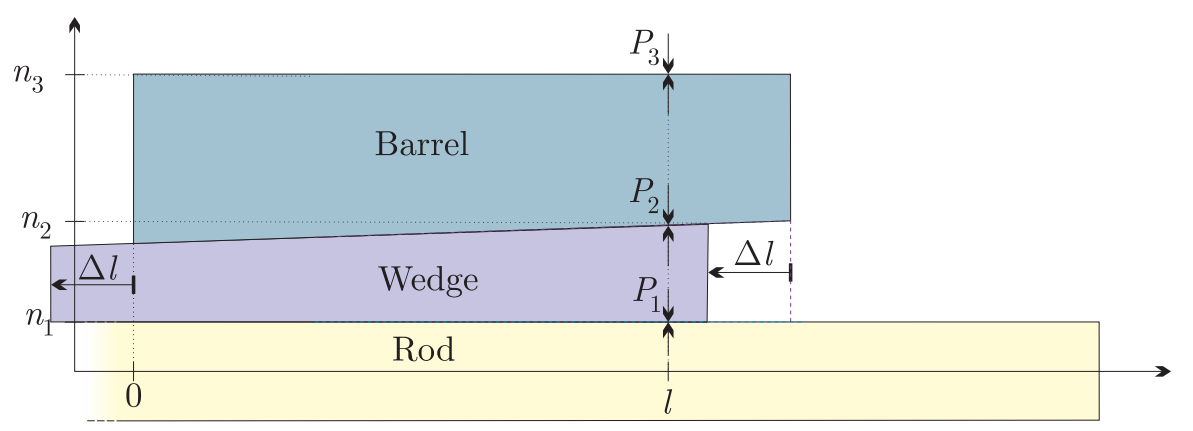

Figure 2: After setting of the wedge with a distance $\Delta l$, we want to compute the new surface radii $n_{k}$ and the the pressures $P_{k}$ at longitudinal position $l$. Note from the setup that $P_{3}=0$. 
3. From equation (1d $)$ and the fact that $n_{3}>0$, we get

$$
n_{3}\left(P_{1}, P_{2}\right)=\sqrt{\frac{E_{\mathrm{b}} r_{\mathrm{bi}}-\left(E_{\mathrm{b}}+P_{2}\left(1-\nu_{\mathrm{b}}\right)\right) n_{2}\left(P_{1}, P_{2}\right)}{E_{\mathrm{b}} r_{\mathrm{bi}}+\left(P_{2}\left(1+\nu_{\mathrm{b}}\right)-E_{\mathrm{b}}\right) n_{2}\left(P_{1}, P_{2}\right)}} n_{2}\left(P_{1}, P_{2}\right) .
$$

4. Equations (1c) and (1e) give the system of equations

$$
\left\{\begin{aligned}
f\left(P_{1}, P_{2}\right) \stackrel{\text { def }}{=} & E_{\mathrm{w}}\left(r_{\mathrm{wo}}-n_{2}\left(P_{1}, P_{2}\right)\right)\left(n_{2}\left(P_{1}, P_{2}\right)^{2}-n_{1}\left(P_{1}\right)^{2}\right) \\
& +\left(2 P_{1} n_{1}\left(P_{1}\right)^{2}-P_{2}\left[\left(1-\nu_{\mathrm{w}}\right) n_{2}\left(P_{1}, P_{2}\right)^{2}+\left(1+\nu_{\mathrm{w}}\right) n_{1}\left(P_{1}\right)^{2}\right]\right) n_{2}\left(P_{1}, P_{2}\right)=0 \\
g\left(P_{1}, P_{2}\right) \stackrel{\text { def }}{=} & E_{\mathrm{b}}\left(r_{\mathrm{bo}}-n_{3}\left(P_{1}, P_{2}\right)\right)\left(n_{3}\left(P_{1}, P_{2}\right)^{2}-n_{2}\left(P_{1}, P_{2}\right)^{2}\right) \\
& +2 P_{2} n_{2}\left(P_{1}, P_{2}\right)^{2} n_{3}\left(P_{1}, P_{2}\right)=0
\end{aligned}\right.
$$

5. A numerical solution of (2d) gives the pressures $P_{1}$ and $P_{2}$ that are inserted in (2a $)-(2 \mathrm{C})$.

As explained in Section 2, of the computed parameters $P_{1}, P_{2}, n_{1}, n_{2}$ and $n_{3}$, the radial stress $P_{1}$ onto the rod is of main interest. Therefore, we will choose to plot $P_{1}$ in our comparisons.

Remark 1. Equations (2b) and (2C) make sense only for $P_{1}$ and $P_{2}$ such that the right-hand sides contain square roots of something positive. Let $\mathcal{P}_{0}$ be the set of all such $\left(P_{1}, P_{2}\right)$. Without any additional information, the solution of (2d) must be expected to be anywhere inside $\mathcal{P}_{0}$, but for numerical algorithms to work, caution is needed to not search outside or even on the boundary of $\mathcal{P}_{0}$, since both $n_{2}$ and $n_{3}$ have singularities at boundary points where the denominators in (2b) or (2c) vanish. Thus, it is necessary to compute $\mathcal{P}_{0}$ in terms of certain somewhat technical but easily implemented conditions on $P_{1}$ and $P_{2}$, which is the topic of Section 4.1, where we compute the subset $\mathcal{P}$ of $\mathcal{P}_{0}$ obtained under an additional restriction (4) to possible values of $P_{1}$.

Remark 2. For the original physical setup in Figure 2, there is of course exactly one unique solution $\left(P_{1}, P_{2}\right)$ for any given $\Delta l$ and $l$. Our derivation of equations (1) in Appendix $\mathbb{A}$ contains only three simplifying assumptions: Axial symmetry, plane stress and linear elasticity. However, this only means to assume a slightly different physical setup that also clearly has exactly one unique solution. This existence and uniqueness is an important underlying assumption in our numerical solution of (2d), so the just described observation saves us from deriving some kind of mathematical proof of the existence of a unique solution of (2d $)$ under the conditions derived in Section 4.1 (or other conditions), which probably would have been a rather challenging task.

\subsection{The set $\mathcal{P}$ containing the solution of (2d) but no singularities}

We summarize some immediate conditions in (3) before choosing an interval for $P_{1}$ in (4) and finally, for $P_{1}$ fixed, express the set $\mathcal{P}$ (defined in Remark $\mathbb{1}$ ) in terms of conditions on $\left(P_{1}\right.$ and) $P_{2}$ in Proposition 13 .

It is clear from the physical setup, geometry and typical material properties that

$$
\begin{gathered}
P_{1}, P_{2} \geq 0, \\
E_{\mathrm{b}}, E_{\mathrm{r}}, E_{\mathrm{w}}, r_{\mathrm{bi}}, r_{\mathrm{bo}}, r_{\mathrm{ro}}, r_{\mathrm{wi}}, r_{\mathrm{wo}}, n_{1}, n_{2}, n_{3}>0, \\
r_{\mathrm{wi}}=r_{\mathrm{ro}}, \\
r_{\mathrm{bi}} \leq n_{2}\left(P_{1}, P_{2}\right) \leq r_{\mathrm{wo}}, \\
r_{\mathrm{bo}} \leq n_{3}\left(P_{1}, P_{2} \leq r_{\mathrm{wo}}+\left(r_{\mathrm{bo}}-r_{\mathrm{bi}}\right),\right. \\
-1 \leq \nu_{\mathrm{r}} \leq 1 / 2, \quad-1 \leq \nu_{\mathrm{w}} \leq 1 / 2 \quad \text { and } \quad-1 \leq \nu_{\mathrm{b}} \leq 1 / 2 .
\end{gathered}
$$


We find an upper bound for $P_{1}$ by observing in figures 2 and 3 that if the wedge is pushed in a distance $\Delta l$, the new radius $n_{1}$ of the rod must certainly have a lower bound

$$
n_{1} \geq n_{1}^{\star} \stackrel{\text { def }}{=} r_{\text {ro }}-\Delta l \tan \left(\delta_{\mathrm{w}}\right) .
$$

Hence, we have from (2a and (3f) that

$$
\begin{aligned}
n_{1}^{\star} & \leq n_{1}=\frac{E_{\mathrm{r}} r_{\mathrm{ro}}}{E_{\mathrm{r}}+P_{1}\left(1-\nu_{\mathrm{r}}\right)} \\
n_{1}^{\star} E_{\mathrm{r}}+n_{1}^{\star} P_{1}\left(1-\nu_{\mathrm{r}}\right) & \leq E_{\mathrm{r}} r_{\mathrm{ro}} \\
P_{1} & \leq \frac{E_{\mathrm{r}}\left(r_{\mathrm{ro}}-n_{1}^{\star}\right)}{n_{1}^{\star}\left(1-\nu_{\mathrm{r}}\right)}
\end{aligned}
$$

For any such $P_{1}$, the following three propositions narrow down the search area to $\mathcal{P}$. All proofs follow in Appendix B, Equations (5) are conditions on $P_{2} / P_{1}$ for (2b) to contain the square root of something positive:

Proposition 1. Suppose that (3) holds and $P_{1}>0$. Then $\frac{n_{2}\left(P_{1}, P_{2}\right)}{n_{1}\left(P_{1}\right)}$ is an increasing function of $\frac{P_{2}}{P_{1}}$ well-defined by $(2 \mathrm{~b})$ if

$$
0 \leq \frac{P_{2}}{P_{1}}<\frac{E_{\mathrm{w}}\left(1-\nu_{\mathrm{r}}\right)+E_{\mathrm{r}}\left(1+\nu_{\mathrm{w}}\right)}{2 E_{\mathrm{r}}} \quad \text { and } \quad E_{\mathrm{w}}>\frac{1-\nu_{\mathrm{w}}}{1-\nu_{\mathrm{r}}} E_{\mathrm{r} .}
$$

Similarly, $\frac{n_{2}\left(P_{1}, P_{2}\right)}{n_{1}\left(P_{1}\right)}$ is a decreasing function of $\frac{P_{2}}{P_{1}}$ well-defined by (2b) if

$$
\frac{P_{2}}{P_{1}}>\frac{E_{\mathrm{w}}\left(1-\nu_{\mathrm{r}}\right)+E_{\mathrm{r}}\left(1+\nu_{\mathrm{w}}\right)}{2 E_{\mathrm{r}}} \quad \text { and } \quad E_{\mathrm{w}}<\frac{1-\nu_{\mathrm{w}}}{1-\nu_{\mathrm{r}}} E_{\mathrm{r}}
$$

In both these cases,

$$
\frac{n_{2}\left(P_{1}, P_{2}\right)}{n_{1}\left(P_{1}\right)}=\sqrt{\left|\frac{E_{\mathrm{w}}\left(1-\nu_{\mathrm{r}}\right)-E_{\mathrm{r}}\left(1-\nu_{\mathrm{w}}\right)}{E_{\mathrm{w}}\left(1-\nu_{\mathrm{r}}\right)+E_{\mathrm{r}}\left(1+\nu_{\mathrm{w}}\right)}\right|} \frac{1}{\sqrt{\left|1-\frac{2 E_{\mathrm{r}}}{\left(E_{\mathrm{w}}\left(1-\nu_{\mathrm{r}}\right)+E_{\mathrm{r}}\left(1+\nu_{\mathrm{w}}\right)\right)} \frac{P_{2}}{P_{1}}\right|}} .
$$

Moreover, if $E_{\mathrm{w}}=\frac{1-\nu_{\mathrm{w}}}{1-\nu_{\mathrm{r}}} E_{\mathrm{r}}$, then $\frac{P_{2}}{P_{1}}=\frac{1}{2} \frac{E_{\mathrm{w}}\left(1-\nu_{\mathrm{r}}\right)+E_{\mathrm{r}}\left(1+\nu_{\mathrm{w}}\right)}{E_{\mathrm{r}}}$ and $\frac{n_{2}\left(P_{1}, P_{2}\right)}{n_{1}\left(P_{1}\right)}$ depends in a more complicated way on the different parameters in (10) (explained in more detail in the proof).

Next, the following condition (7) guarantees that also (2c) contains the square root of something positive.

Proposition 2. Suppose that (5a) or (5b) holds. Under the constraints (3), for $n_{3}$ to be

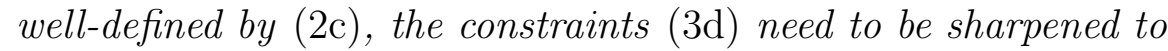

$$
0<P_{2}<\frac{E_{\mathrm{b}}}{1+\nu_{\mathrm{b}}} \quad \text { and } \quad \frac{E_{\mathrm{b}} r_{\mathrm{bi}}}{E_{\mathrm{b}}-P_{2}\left(1+\nu_{\mathrm{b}}\right)}<n_{2}\left(P_{1}, P_{2}\right) \leq r_{\text {wo }}
$$
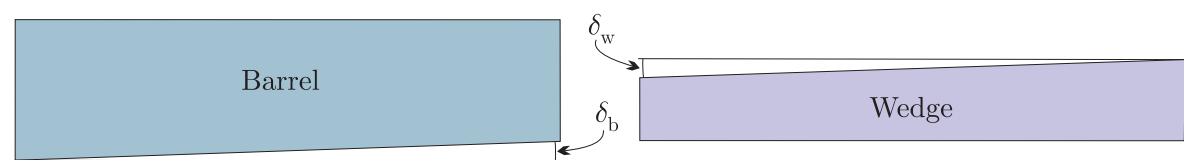

Figure 3: The barrel and wedge cross-sections in Figure 2 have different angles $\delta_{\mathrm{b}}$ and $\delta_{\mathrm{w}} \geq \delta_{\mathrm{b}}$ before the setting of the wedge. 
Finally, we can reformulate (7) into the following bounds only on $P_{2}$ :

Proposition 3. Suppose that (3) holds and $P_{1}>0$. Define

$$
\begin{gathered}
h\left(P_{1}\right) \stackrel{\text { def }}{=} \frac{E_{\mathrm{w}}\left(1-\nu_{\mathrm{r}}\right)-E_{\mathrm{r}}\left(1-\nu_{\mathrm{w}}\right)}{E_{\mathrm{b}}^{2} r_{\mathrm{bi}}^{2}\left(E_{\mathrm{r}}+P_{1}\left(1-\nu_{\mathrm{r}}\right)\right)^{2}} E_{\mathrm{r}} r_{\mathrm{ro}}^{2} P_{1}, \\
a\left(P_{1}\right) \stackrel{\text { def }}{=} \frac{1}{h\left(P_{1}\right)\left(1+\nu_{\mathrm{b}}\right)^{2}}-\frac{E_{\mathrm{b}}}{1+\nu_{\mathrm{b}}} \quad \text { and } \quad b\left(P_{1}\right) \stackrel{\text { def }}{=} \frac{E_{\mathrm{b}}^{2}}{\left(1+\nu_{\mathrm{b}}\right)^{2}}-\frac{E_{\mathrm{w}}\left(1-\nu_{\mathrm{r}}\right)+E_{\mathrm{r}}\left(1+\nu_{\mathrm{w}}\right)}{E_{\mathrm{r}} h\left(P_{1}\right)\left(1+\nu_{\mathrm{b}}\right)^{2}} P_{1} .
\end{gathered}
$$

Then $n_{3}$ is well-defined by (2C) if and only if (5a) is sharpened to the following bounds on $P_{2}$ :

1. If $E_{\mathrm{w}}>\frac{1-\nu_{\mathrm{w}}}{1-\nu_{\mathrm{r}}} E_{\mathrm{r}}$, then

$0<P_{2}<\min \left(\frac{E_{\mathrm{b}}}{1+\nu_{\mathrm{b}}},\left(\frac{E_{\mathrm{w}}\left(1-\nu_{\mathrm{r}}\right)+E_{\mathrm{r}}\left(1+\nu_{\mathrm{w}}\right)}{E_{\mathrm{r}}}-\frac{E_{\mathrm{w}}\left(1-\nu_{\mathrm{r}}\right)-E_{\mathrm{r}}\left(1-\nu_{\mathrm{w}}\right)}{\left(E_{\mathrm{r}}+P_{1}\left(1-\nu_{\mathrm{r}}\right)\right)^{2}} E_{\mathrm{r}} \frac{r_{\mathrm{ro}}^{2}}{r_{\mathrm{wo}}^{2}}\right) \frac{P_{1}}{2}\right)$.

Moreover, for the polynomial $\mathcal{P}_{P_{1}}(x) \stackrel{\text { def }}{=} x^{2}+2 a\left(P_{1}\right) x+b\left(P_{1}\right)$, it must also hold that

$$
\begin{array}{lll}
\mathcal{P}_{P_{1}}\left(-a\left(P_{1}\right)\right)>0 & \text { or } & P_{2}<-a\left(P_{1}\right)-\sqrt{a\left(P_{1}\right)^{2}-b\left(P_{1}\right)} \\
& \text { or } & P_{2}>-a\left(P_{1}\right)+\sqrt{a\left(P_{1}\right)^{2}-b\left(P_{1}\right)} .
\end{array}
$$

2. If $E_{\mathrm{w}}<\frac{1-\nu_{\mathrm{w}}}{1-\nu_{\mathrm{r}}} E_{\mathrm{r}}$, then (9b) must hold and

$$
\left(\frac{E_{\mathrm{w}}\left(1-\nu_{\mathrm{r}}\right)+E_{\mathrm{r}}\left(1+\nu_{\mathrm{w}}\right)}{E_{\mathrm{r}}}-\frac{E_{\mathrm{w}}\left(1-\nu_{\mathrm{r}}\right)-E_{\mathrm{r}}\left(1-\nu_{\mathrm{w}}\right)}{\left(E_{\mathrm{r}}+P_{1}\left(1-\nu_{\mathrm{r}}\right)\right)^{2}} E_{\mathrm{r}} \frac{r_{\mathrm{ro}}^{2}}{r_{\mathrm{wo}}^{2}}\right) \frac{P_{1}}{2}<P_{2}<\frac{E_{\mathrm{b}}}{1+\nu_{\mathrm{b}}} .
$$

3. If $E_{\mathrm{w}}=\frac{1-\nu_{\mathrm{w}}}{1-\nu_{\mathrm{r}}} E_{\mathrm{r}}$, then $\frac{P_{2}}{P_{1}}=\frac{1}{2} \frac{E_{\mathrm{w}}\left(1-\nu_{\mathrm{r}}\right)+E_{\mathrm{r}}\left(1+\nu_{\mathrm{w}}\right)}{E_{\mathrm{r}}}$.

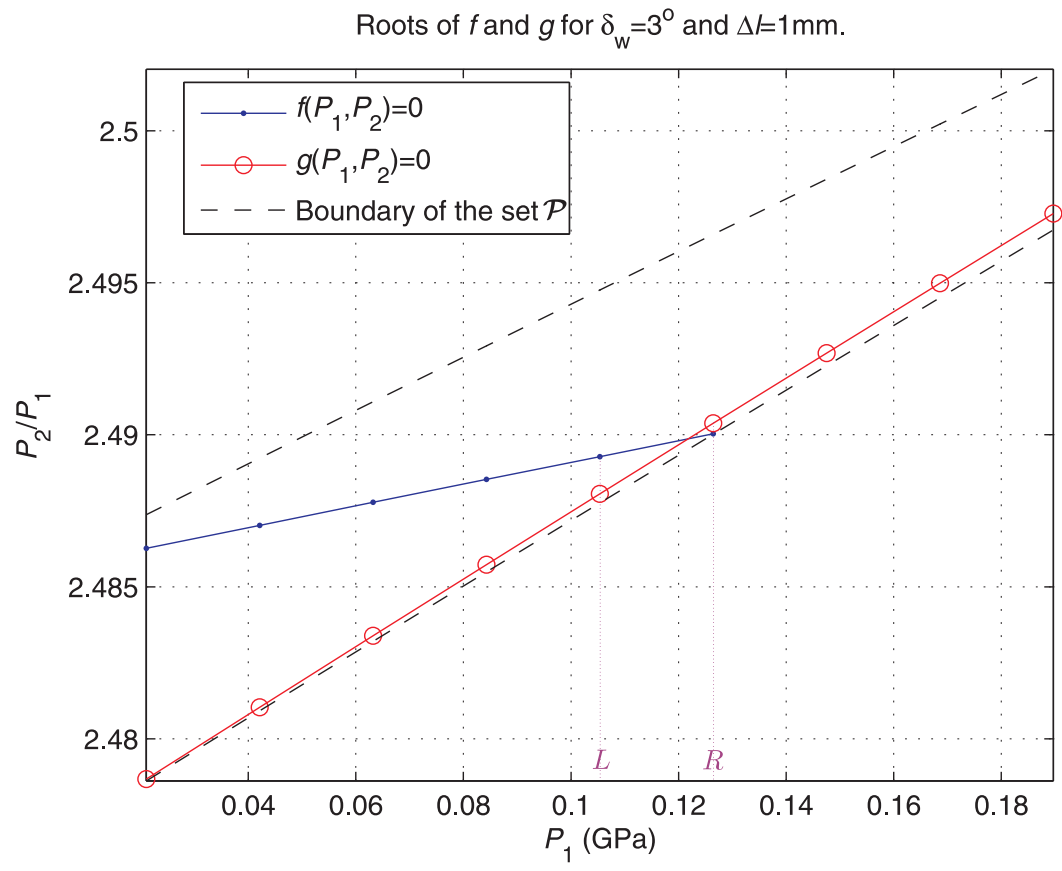

Figure 4: For fixed $P_{1}$, zeros of $f$ and $g$ are found by using the bisection method in the interval specified in Propositions 13. 


\subsection{The algorithm and numerical results}

We have now computed the borderlines of a set $\mathcal{P}$ big enough to contain the unique solution of equations (2) and small enough for all the singularities of $n_{2}$ and $n_{3}$ to be on the boundary of $\mathcal{P}$ but not in $\mathcal{P}$, thus guaranteeing that the analyzed functions (2) are continuous on $\mathcal{P}$. For different fixed $P_{1}$, our numerical solution of $(2 \mathrm{~d})$ is based on computing separate solutions of $f=0$ and $g=0$ using the bisection method. This is possible because $f$ and $g$ as functions of $P_{2}$ always attain both positive and negative values and both have one unique zero-crossing. This is most easily understood from a physical interpretation of equations (2) for any fixed $\left(P_{1}, P_{2}\right)$ in $\mathcal{P}$ :

1. If radial pressure $P_{1}$ is applied to a rod with radius $r_{\text {ro }}$, then (2a) tells the resulting new radius $n_{1}\left(P_{1}\right)$. (Via (18b) and Figure 12 in the proofs.)

2. Choose a wedge with inner radius $r_{\mathrm{wi}}=r_{\mathrm{ro}}$ and with outer radius (at longitudal distance l) $\widetilde{r_{\mathrm{wo}}}=\widetilde{r_{\mathrm{wo}}}\left(P_{1}, P_{2}, r_{\mathrm{wi}}\right)$ such that applying the radial pressures $P_{1}$ and $P_{2}$ to the inner and outer surfaces, respectively, gives the new inner radius $n_{1}\left(P_{1}\right)$. Then (2b) gives the resulting new outer radius $n_{2}\left(P_{1}, P_{2}\right)$. (Via (18d) and Figure 13 in the proofs.)

3. Next choose a barrel with inner radius $r_{\mathrm{bi}}$ and outer radius $\widetilde{r_{\mathrm{bo}}}=\widetilde{r_{\mathrm{bo}}}\left(P_{1}, P_{2}, r_{\mathrm{bi}}\right)$ such that applying radial pressure $P_{2}$ to the inner surface gives the new inner radius $n_{2}\left(P_{1}, P_{2}\right)$. Then (2C) gives the new resulting outer radius $n_{3}\left(P_{1}, P_{2}\right)$. (Via (18h) and Figure 14 in the proofs.)

4. Finally, equations (2d) are exactly the conditions on $P_{1}, P_{2}$ that give $\widetilde{r_{\text {wo }}}=r_{\text {wo }}$ and $\widetilde{r_{\mathrm{bo}}}=r_{\mathrm{bo}}$. (Via equations (18f), (181) and figures 13 14)

For only $P_{1}$ fixed, $\widetilde{r_{\text {wo }}}=r_{\text {wo }}$ corresponds to choosing $P_{2}$ such that $f\left(P_{1}, P_{2}\right)=0$. From item 2 in this list we understand that this $P_{2}$ is unique and that with small adjustments of it, we can get both $\widetilde{r_{\text {wo }}}<r_{\text {wo }}$ and $\widetilde{r_{\text {wo }}}>r_{\text {wo }}$, corresponding to $f>0$ and $f<0$, respectively.

Exactly the same reasoning for item 3 and $\widetilde{r_{\text {bo }}}$ gives that for $P_{1}$ fixed, there is a unique $P_{2}$ giving $g=0$ and small adjustments of this $P_{2}$ gives $g<0$ and $g>0$, respectively.

Figure 4 shows the boundary of $\mathcal{P}$ as well as the the lines $f\left(P_{1}, P_{2}\right)=0$ and $g\left(P_{1}, P_{2}\right)=0$ for one of the investigated choices of input parameters. Note that the sought solution, the crossing of those two lines, is quite close to the singularities at the boundary. This is why it is important to know the exact location of this boundary, which we derived in propositions 1 3 . Note also that the lines $f\left(P_{1}, P_{2}\right)=0$ and $g\left(P_{1}, P_{2}\right)=0$ not always are inside $\mathcal{P}$. Hence one important step in the following algorithm is to strengthen the condition (4) to restrict $P_{1}$ to a smaller interval $[L, R]$ inside which both of the lines crosses and are in $\mathcal{P}$, such as the choice of $[L, R]$ indicated in Figure 4. Once that is done, a bisection approach can be used on the interval $[L, R]$ until it is short enough to give the solution of $f\left(P_{1}, P_{2}\right)=g\left(P_{1}, P_{2}\right)=0$ with the desired numerical precision. Thus we get the following

\section{Algorithm}

1. For $N$ different and equally spaced choices of $P_{1}$ in the interval given by equations (4), do the following $(N=10$ in our implementation).

(a) Compute the endpoints of the intervals of all $P_{2}$ such that $\left(P_{1}, P_{2}\right)$ is in $\mathcal{P}$, as given by Proposition 3 . 


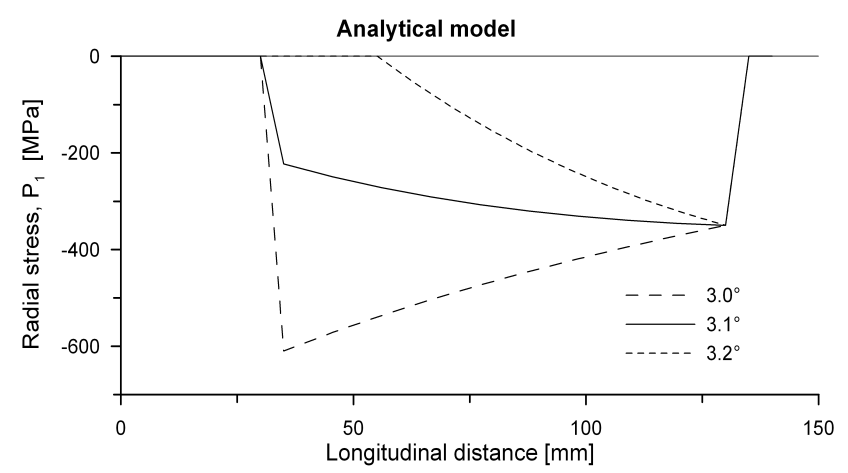

(a)

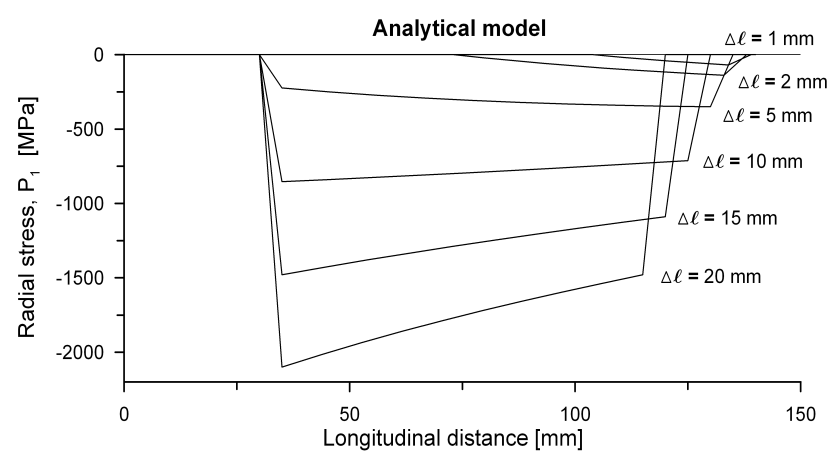

(b)

Figure 5: The dependence of $P_{1}$ on $l$ with the analytical model of Section 4. (a) Various $\delta_{\mathrm{w}}$ and $\Delta l=5$. (b) Various $\Delta l$ and $\delta_{\mathrm{w}}=3.1^{\circ}$.

(b) For $u\left(P_{2}\right)=f\left(P_{1}, P_{2}\right)$ and $u\left(P_{2}\right)=g\left(P_{1}, P_{2}\right)$ (as defined in $(2 \mathrm{~d}$ ) $)$, do the following: If $u$ has different sign at the endpoints of one of the just computed intervals (or rather immediately inside the endpoints, say, as close to potential borderline singularities as machine precision allows, see BGS10), then $u$ has a zero inside $\mathcal{P}$. In that case, use the bisection method on $P_{2}$ to solve the corresponding equation $u=0$.

2. If necessary, repeat step 1 for additional (denser sampled) values of $P_{1}$ until a subinterval $[L, R]$ is found such that both $f(L, \cdot), f(R, \cdot), g(L, \cdot)$ and $r(R, \cdot)$ have zerocrossings inside $\mathcal{P}$ and such that the lines $f\left(P_{1}, P_{2}\right)=0$ and $g\left(P_{1}, P_{2}\right)=0$ cross for some $P_{1}$ in $[L, R]$, that is, $L$ and $R$ must be such that there are zero crossings

$$
f\left(L, a_{L}\right)=g\left(L, b_{L}\right)=f\left(R, a_{R}\right)=g\left(R, b_{R}\right)=0,
$$

such that $a_{L}-b_{L}$ and $a_{R}-b_{R}$ have different signs.

3. Continue repeating step 1 with $P_{1}$ restricted to shorter and shorter such intervals $[L, R]$ until the joint zero of $f$ and $g$ is found with desired numerical precision.

For a complete and fully detailed description of this algorithm, a MATLAB implementation is included and described in BGS10.

\section{Numerical results}

For the radial and circumferential direction material parameters listed in Table 1, $\frac{1-\nu_{\mathrm{w}}}{1-\nu_{\mathrm{r}}} E_{\mathrm{r}}=$ $\frac{1-0.34}{1-0.3} * 10<9.43<70=E_{\mathrm{w}}$, so only conditions (4) and (9) are needed to compute the results presented in this paper. Figure 5 shows the longitudinal variation of the radial stress onto the rod computed with our MATLAB implementation of the just described algorithm. Figure 5 (a) shows the dependence of $P_{1}$ on $l$ for a constant presetting distance of $\Delta l=5 \mathrm{~mm}$ and various angles on the wedge's outer surface. Similarly, Figure 5 (b) shows the corresponding pressures for six different presetting distances $\Delta l$ with a given wedge angle $\delta_{\mathrm{w}}=3.1^{\circ}$. All of these plots show that the analytical model transfers more stress towards the back of the anchorage when the difference in the angle between the barrel's inner surface and the wedge's outer surface increases. The model also predicts an increase in overall radial stress with increased presetting distance. Both of these results are expected.

\section{Axi-symmetric FEM}

To verify the analytical results, a FE-model with the same material and geometrical properties was created. The difference is the assumption of plane stress in the analytical solution; in 


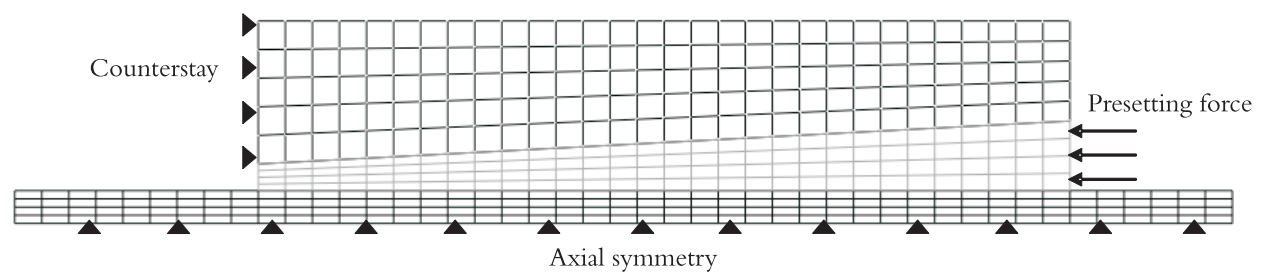

Figure 6: Axi-symmetric FE-model.

the FE-model no such simplification is included. In the former, the wedge to be pushed into the barrel can instead be seen as growing in the radial direction, though it is positioned at the same longitudinal position. It can be seen as a structure that is infinitely short in the longitudinal direction with a growing radial size of the wedge, thus allowing for the materials to expand freely in the longitudinal direction. The FE-model is on the other hand modeled with the complete wedge pushed into the barrel. This creates longitudinal stresses that somewhat counteract the longitudinal expansion of the materials as they are compressed in the radial direction. Moreover, as discussed in Section 3.1.3, our FE-models also utilize the orthotropic properties of the CFRP rod.

\subsection{Model}

The model is created and analysed with a nonlinear geometry analysis in Abaqus Standard 6.7-1. Geometry, element distribution, boundary conditions and application of the presetting force are seen in Figure 6. All elements are of the type "CAX4R", i.e. a 4-node axisymmetric quadrilateral element with reduced integration and hourglass control. In the rod-wedge interface, rough frictional behaviour is applied, which here can be assumed to be a tied behaviour. In the wedge-barrel interface, a frictionless interaction is applied. Setting the longitudinal motion of the barrels front end to zero throughout the loading provides counteraction. Prescribing a linearly increasing intrusion of the wedge into the anchorage provides the presetting. Three different wedge angles are used, $3.0^{\circ}, 3.1^{\circ}$ and $3.2^{\circ}$, and the final intrusion of the wedge is set to $5 \mathrm{~mm}$.

\section{$5.2 \quad$ Results}

For a fixed inner barrel angle of $3.0^{\circ}$ and wedges with varying outer angles, the axisymmetric FE-model gives the radial stresses shown in Figure 7 (a). When the wedge has the same angle as the barrel, $3.0^{\circ}$, the highest radial stresses appear at the front of the anchorage, where the wedge has its thin end. Through the increasing difference in angle, less stress is experienced at the front of the anchorage. When the difference is $0.2^{\circ}$ no stress can be detected in the first 20 $\mathrm{mm}$ of the anchorage. For all cases plotted in Figure 7 , the stresses onto the rod at the back of the anchorage are similar in amplitude.

\section{$6 \quad 3 \mathrm{D}$ FEM}

3D models are more complicated to create and require higher computational costs. By also finding symmetry in these cases, the workload may be lowered several times. In this case, with the axisymmetric rod and barrels and three wedges with three spacings in between, the model can be reduced to $1 / 6$ of its original size. 


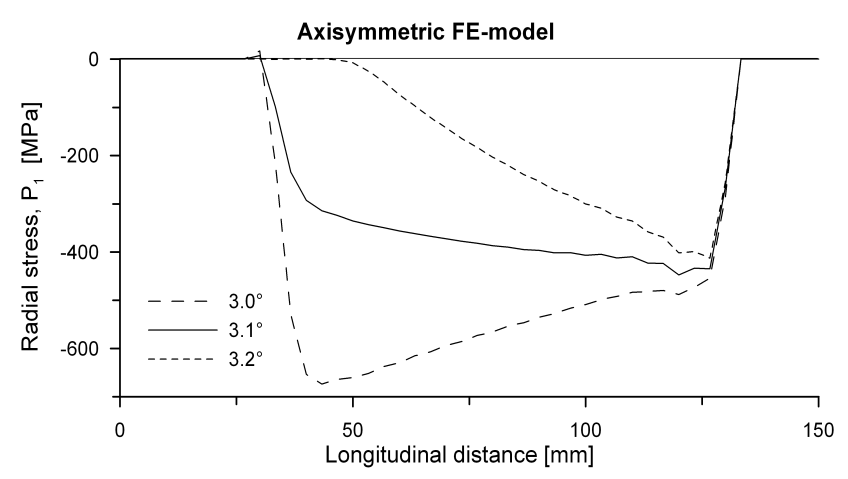

(a)

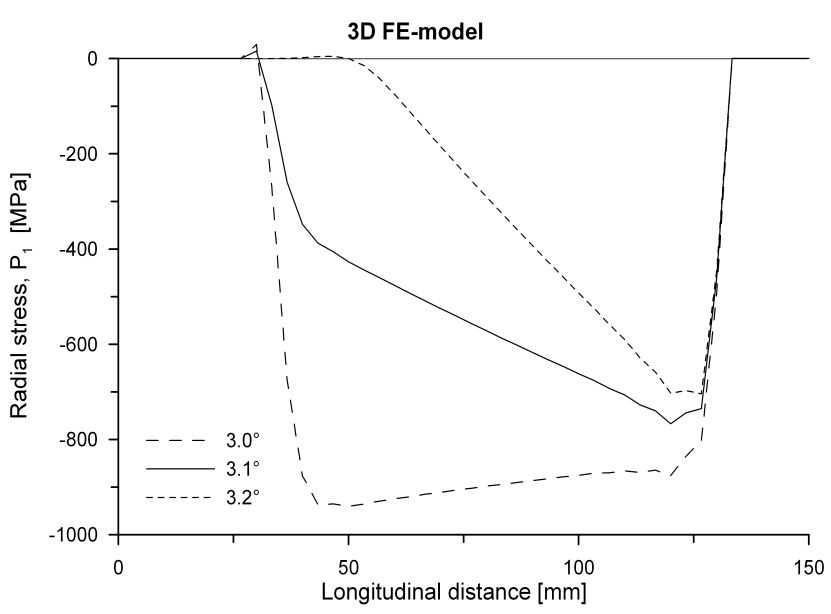

(b)

Figure 7: Radial stress $P_{1}$ along the rod for three different wedge outer angles $\delta_{\mathrm{w}}$. FEM results. (a) Axisymmetric FEM. (b) 3D FEM.

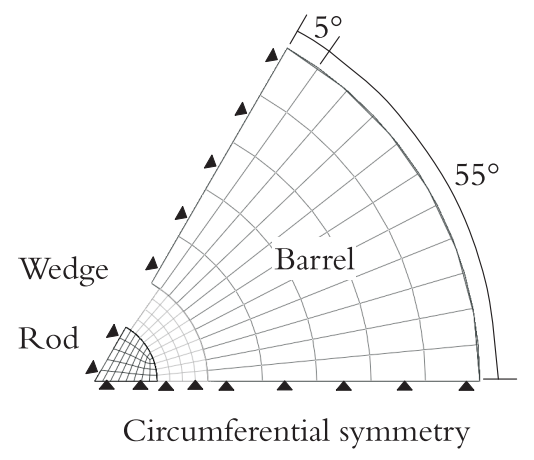

Figure 8: Front view of the 3D model.

\subsection{Model}

The minimized 3D model is achieved by one cut along the anchorage at the centre of one wedge and one cut at the centre of an adjacent spacing. Along the created surfaces no displacement is allowed perpendicular to the surfaces. All nodes are allowed to displace in the longitudinal and radial directions, thus creating a set of boundary conditions identical to the axi-symmetric case. Figure 8 shows the created model. Table 1 presents the remaining geometries and material properties used. Elements used are of the type "C3D8R", i.e. an 8 node linear brick element with reduced integration and hourglass control. The application of loads, frictional behaviours, nonlinear geometry and variations of differences in angle are identical to the cases described for the axi-symmetric model.

\subsection{Results}

To receive a correct value of the radial stress in each section along the rod, which can be compared with the values from the analytical solution and the axi-symmetric FE- model, an average of the stresses in the radial direction must be calculated. This value is based upon the 11 nodes on the rod, which in each longitudinal section are in contact with the wedge. Accordingly, the only free node is not included. Figure 7 (b) shows the average pressures for the three different outer angles of the wedges used in the analysis. 


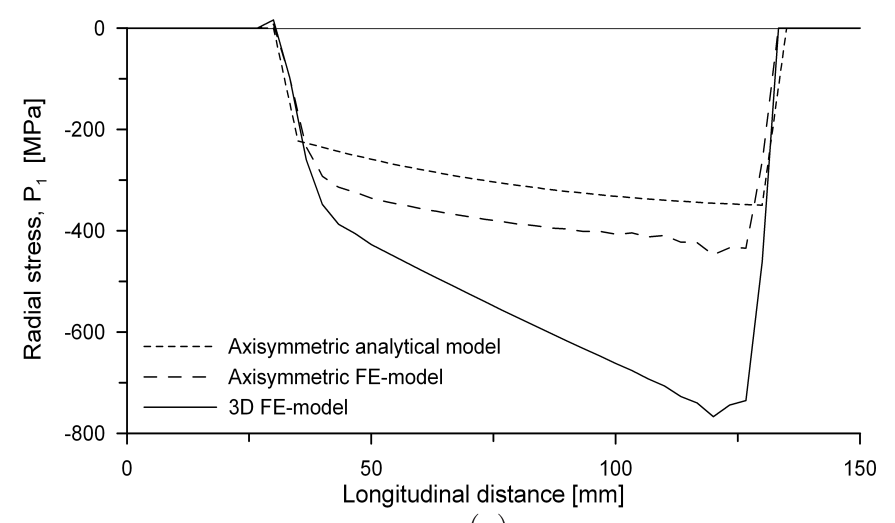

(a)

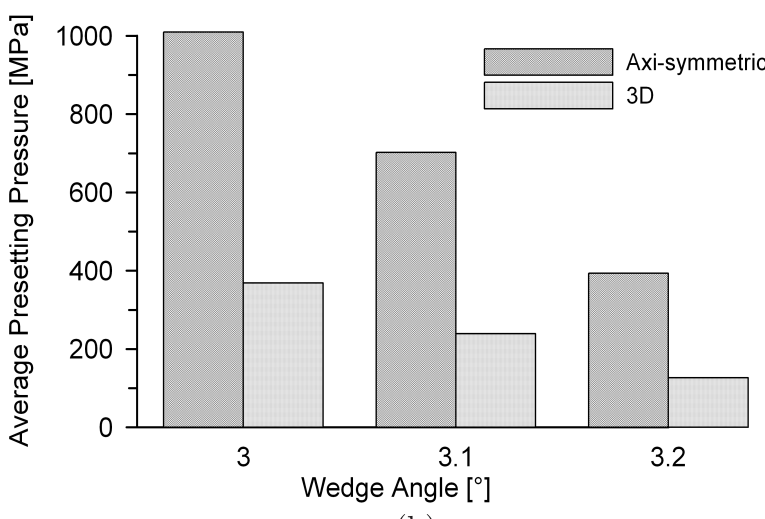

(b)

Figure 9: (a) Comparison of the examined models for $\Delta l=5 \mathrm{~mm}$ and $\delta_{\mathrm{w}}=3.1^{\circ}$. (b) Pressure applied to the back of the wedge for $\Delta l=5 \mathrm{~mm}$ in the different FE-models.

\section{Results and discussion}

Figure 9 (a) shows the longitudinal distribution of radial stresses onto the CFRP rod for the analytical axi-symmetric model, the FE axi-symmetric model and the FE 3D model. In this Figure, $\Delta l$ is fixed at $5 \mathrm{~mm}$ and the outer angle $\delta_{\mathrm{w}}$ of the wedge is $3.1^{\circ}$. For this longitudinal distribution, there is a distinct correspondence between the analytical model and the axi-symmetric numerical model, but also a small difference in amplitude. This difference is likely due to the plane stress assumption that our analytical model relies on, which removes the longitudinal confinement (see Figure 11, Appendix Ad) and thus a substantial part of the radial pressure. Contradictory to this, AMSP01a, AMSP07 present results where the radial pressure is higher in the analytical model than the axi-symmetric FE-model. The assumptions in the models are to some extent different between the models presented therein and in this paper. While the models in this paper use elastic properties for all materials, AMSP01a, AMSP07 use plastic properties for an inner sleeve positioned between the rod and the wedges. It should also be noted that the wedges in AMSP01a, AMSP07 are made by steel and that the geometry has been altered in AMSP07. It seems as if the results are highly dependent on the assumptions made in the creation of the models and that a perfect correlation in the shape of the pressure distribution as well as the size of it might be difficult to achieve.

When comparing the axi-symmetric models to the numerical 3D model, and to the offset most likely caused by the plane stress assumption, there is now a bigger difference in both the shape and amplitude of the stress distribution curve. The 3D model produces stronger radial stresses onto the rod than the axi-symmetric models, and shows a higher rate of increase of the radial stress $P_{1}$ towards the back of the anchorage. We believe that these differences are due to the lack of circumferential confinement for the wedges in the 3D-model. This lack of confinement reduces the capacity to carry stresses through arching, which becomes more pronounced where the material is thicker and thus capable of carrying larger stresses in the circumferential direction when axial symmetry is assumed. In AMSP07 this lack of correspondence is overcome by making the barrel infinitely stiff in the analytical model, thus forcing the wedges to deform inwards and create a higher pressure onto the rod. By doing so they reach an agreement between the models which can not be found if the models are created with the same geometrical and mechanical assumptions.

In Figure 9 (b), note that although the axi-symmetric model results in less radial pressure onto the rod, the energy put into the system is higher. The values are calculated as a weighted average on the longitudinal pressure experienced by the back of the wedge, as it has been pushed $5 \mathrm{~mm}$ into the barrel. It is also noted that a $5 \mathrm{~mm}$ slip requires more force if the inner surface 
of the barrel and the outer surfaces of the wedges are aligned than if they are manufactured with a small difference in angle.

\section{Conclusions}

Based upon results from the analytical and numerical models the following conclusions can be made:

- In the numerical solution of the system of equations from the derived analytical model, the main numerical complication was the need to find the roots (zeros) of two functions rather close to singularities of the same functions. However, after computing the exact location of the boundary containing these singularities, finding the roots using, for example, the bisection method was a rather straightforward task.

- The derived analytical model predicts the behaviour of an axi-symmetric anchorage well up to an offset that is most likely caused by the underlying plane stress assumption. Hence, the analytical model can be a useful and faster alternative to the axi-symmetric FE model when searching for an anchorage design that gives the desired distribution of the pressures $P_{1}$ on the rod.

- The axi-symmetric and 3D models give longitudinal distributions of the radial stresses that differ both in the shape and amplitude of this distribution. Therefore, neither the analytical nor the FE axi-symmetric model can accurately describe the 3D behavior of a conical wedge anchorage.

- Axi-symmetric representations of the conical wedge anchorage require a larger presetting force to set $5 \mathrm{~mm}$ compared to 3D models. A similar observation is made concerning the difference in angle, where a larger difference requires less presetting force.

\section{Acknowledgements}

This paper is based upon work carried out within a project funded by the Development Fund of the Swedish Construction Industry (SBUF) together with Skanska AB. The authors would also like to thank Prof. Björn Täljsten for all fruitful discussions that eventually led to the developed model and the interesting results. 


\section{A Deriving equations (1)}

\section{A.1 The mathematical model}

Figure 10 (a) shows the forces acting on an infinitesimal element. Due to symmetry, forces acting in the longitudinal $(l)$ and circumferential $(\theta)$ directions are of equal size on opposite surfaces. In a plane stress scenario, $\sigma_{1}, \tau_{l r}$ and $\tau_{l \theta}$ are of the same size at the opposite surfaces, see Figure 11 (c). This is the approximation of the actual setup in Figure 11 (a) that we will use. Note, however that the plane strain assumption depicted in Figure 11 (b) in fact results in exactly the same mathematical model with only some modifications to a few material constants, as explained in Remark 3, page 20. Thus this appendix does in fact treat both those scenarios simultaneously.

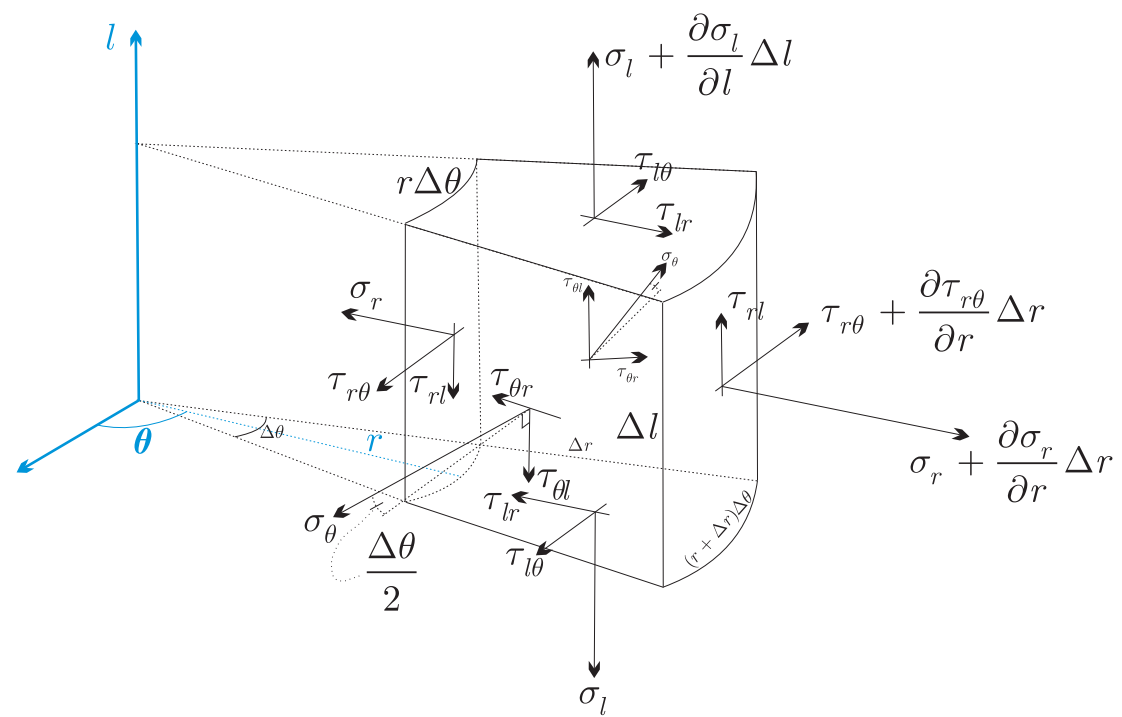

(a)

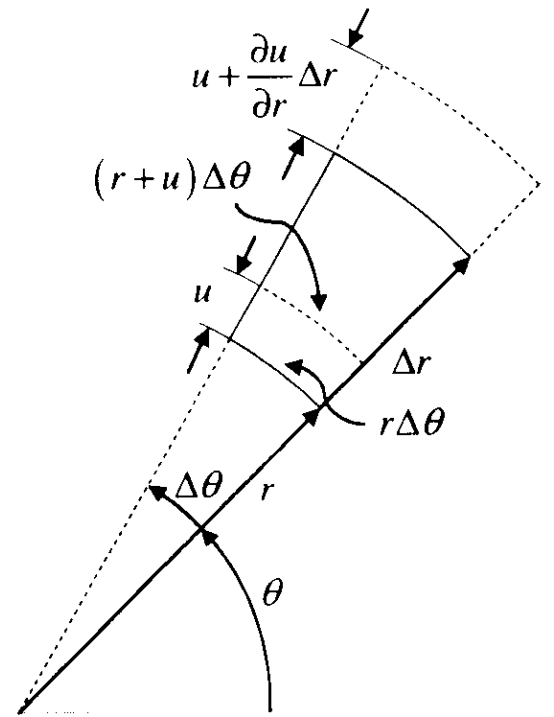

(b)

Figure 10: (a) Stress forces $\sigma_{x}$ and shear forces $\tau_{x y}$ acting on an infinitesimal 3-element,with $x$ denoting the surface and $y$ the direction. (b) Displacement of an infinitesimal element in a state of rotational symmetry.

The actual setup:

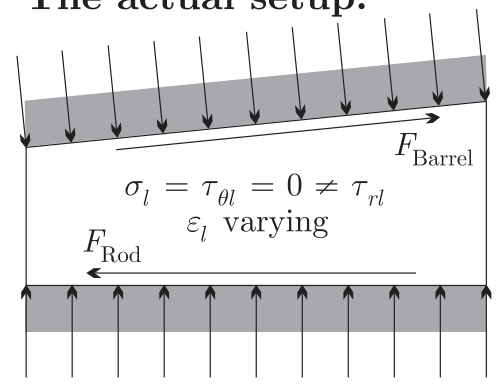

(a)
Plane strain:

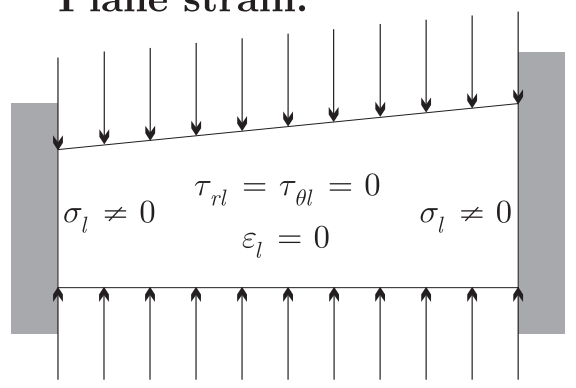

(b)
Plane stress:

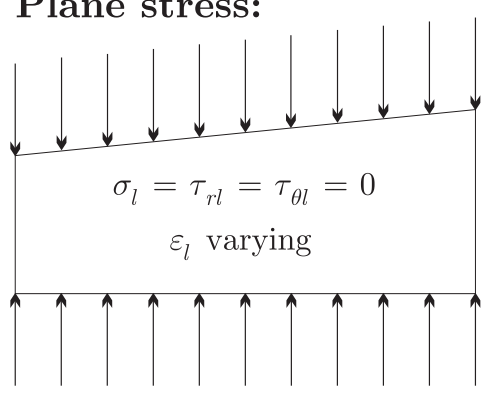

(c)

Figure 11: (a) The forces acting on a cross section of the wedge. (b) One simplification of this scenario is to assume the wedge as fixed between two walls and subjected to forces only in the radial direction so that the strain $\varepsilon_{1}=0$ and $\sigma_{1}$ is nonzero and varying. This is called plane strain. (c) Another simplified scenario is the plane stress scenario with unrestricted expansion in the longitudinal direction and uniform force distribution in the radial direction such that $\varepsilon_{1}$ is varying and $\sigma_{1}=\tau_{r l}=\tau_{\theta l}=0$. 
By summing all forces in radial direction, we get

$$
\begin{aligned}
0=\sigma_{\mathrm{r}} \Delta l \cdot r \Delta \theta & -\left(\sigma_{\mathrm{r}}+\frac{\partial \sigma_{\mathrm{r}}}{\partial r} \Delta r\right) \Delta l \cdot(r+\Delta r) \Delta \theta+2 \sigma_{\theta} \sin \left(\frac{\Delta \theta}{2}\right) \Delta l \Delta r \\
& +\tau_{\theta r} \cos \left(\frac{\Delta \theta}{2}\right) \Delta l \Delta r-\tau_{\theta r} \cos \left(\frac{\Delta \theta}{2}\right) \Delta l \Delta r \\
& +\tau_{l r} \Delta r \frac{r \Delta \theta+(r+\Delta r) \Delta \theta}{2}-\tau_{l r} \Delta r \frac{r \Delta \theta+(r+\Delta r) \Delta \theta}{2} \\
=\quad \sigma_{\mathrm{r}} \Delta l r \Delta \theta & -\left(\sigma_{\mathrm{r}}+\frac{\partial \sigma_{\mathrm{r}}}{\partial r} \Delta r\right) \Delta l(r+\Delta r) \Delta \theta+2 \sigma_{\theta} \sin \left(\frac{\Delta \theta}{2}\right) \Delta l \Delta r .
\end{aligned}
$$

Division by $\Delta l \Delta \theta$ gives

$$
\begin{aligned}
& \sigma_{\mathrm{r}} r-\left(\sigma_{\mathrm{r}}+\frac{\partial \sigma_{\mathrm{r}}}{\partial r} \Delta r\right)(r+\Delta r)+\sigma_{\theta} \frac{\sin \left(\frac{\Delta \theta}{2}\right)}{\frac{\Delta \theta}{2}} \Delta r=0, \\
& -\sigma_{\mathrm{r}} \Delta r-\frac{\partial \sigma_{\mathrm{r}}}{\partial r} \Delta r(r+\Delta r)+\sigma_{\theta} \frac{\sin \left(\frac{\Delta \theta}{2}\right)}{\frac{\Delta \theta}{2}} \Delta r=0, \quad \text { (Divide by } r \Delta r \text { ) } \\
& \frac{\sigma_{\mathrm{r}}}{r}+\frac{\partial \sigma_{\mathrm{r}}}{\partial r}\left(1+\frac{\Delta r}{r}\right)-\frac{\sigma_{\theta}}{r} \frac{\sin \left(\frac{\Delta \theta}{2}\right)}{\frac{\Delta \theta}{2}}=0, \quad(\text { Let }(\Delta r, \Delta \theta) \rightarrow(0,0)) \\
& \frac{\partial \sigma_{\mathrm{r}}}{\partial r}+\frac{\sigma_{\mathrm{r}}-\sigma_{\theta}}{r}=0
\end{aligned}
$$

For $l$ fixed we will now derive the formula (17) below for the radial displacement $u=u(r, l)$ of an infinitesimal element, with positive sign denoting displacements away from the centre, as indicated in Figure 10(b). The resulting radial and circumferential strains $\varepsilon_{\mathrm{r}}$ and $\varepsilon_{\theta}$ at radius $r+u(r, l)$ are defined as the change in length divided by the total length:

$$
\varepsilon_{\mathrm{r}}=\frac{\left(u+\frac{\partial u}{\partial r} \Delta r\right)-u}{\Delta r}=\frac{\partial u}{\partial r} \quad \text { and } \quad \varepsilon_{\theta}=\frac{(r+u) \Delta \theta-r \Delta \theta}{r \Delta \theta}=\frac{u}{r}
$$

These are connected to the stresses $\sigma_{\mathrm{r}}, \sigma_{\theta}$ and $\sigma_{\mathrm{l}}$ at radius $r+u(r, l)$ via Hooke's generalized law

$$
\varepsilon_{\mathrm{r}}=\frac{\sigma_{\mathrm{r}}-\nu\left(\sigma_{\theta}+\sigma_{\mathrm{l}}\right)}{E}, \quad \varepsilon_{\theta}=\frac{\sigma_{\theta}-\nu\left(\sigma_{\mathrm{r}}+\sigma_{\mathrm{l}}\right)}{E} \quad \text { and } \quad \varepsilon_{\mathrm{l}}=\frac{\sigma_{\mathrm{l}}-\nu\left(\sigma_{\mathrm{r}}+\sigma_{\theta}\right)}{E}
$$

with $E$ being Young's modulus and $\nu$ the Poisson ratio. Under the assumption of plane stress, $\sigma_{1}=0$, so that

$$
\varepsilon_{\mathrm{r}}=\frac{\sigma_{\mathrm{r}}-\nu \sigma_{\theta}}{E}, \quad \varepsilon_{\theta}=\frac{\sigma_{\theta}-\nu \sigma_{\mathrm{r}}}{E} \quad \text { and } \quad \varepsilon_{\mathrm{l}}=-\frac{\nu\left(\sigma_{\mathrm{r}}+\sigma_{\theta}\right)}{E}
$$

Insertion of (12) in (13) gives

$$
\left\{\begin{array} { c } 
{ \frac { \partial u } { \partial r } = \frac { \sigma _ { \mathrm { r } } - \nu \sigma _ { \theta } } { E } , } \\
{ \frac { u } { r } = \frac { \sigma _ { \theta } - \nu \sigma _ { \mathrm { r } } } { E } , }
\end{array} \quad \text { or equivalently, } \quad \left\{\begin{array}{c}
\sigma_{\mathrm{r}}=\frac{E}{1-\nu^{2}}\left(\frac{\partial u}{\partial r}+\nu \frac{u}{r}\right) \\
\sigma_{\theta}=\frac{E}{1-\nu^{2}}\left(\frac{u}{r}+\nu \frac{\partial u}{\partial r}\right)
\end{array}\right.\right.
$$


where $u=u(r, l), \sigma_{\mathrm{r}}=\sigma_{\mathrm{r}}(r+u(r, l), l)$ and $\sigma_{\theta}=\sigma_{\theta}(r+u(r, l), l)$. Insertion in (11) gives

$$
\begin{aligned}
0 & =\frac{E}{1-\nu^{2}} \frac{\partial}{\partial r}\left(\frac{\partial u}{\partial r}+\nu \frac{u}{r}\right)+\frac{\frac{E}{1-\nu^{2}}\left(\frac{\partial u}{\partial r}+\nu \frac{u}{r}\right)-\frac{E}{1-\nu^{2}}\left(\frac{u}{r}+\nu \frac{\partial u}{\partial r}\right)}{r} \\
0 & =\frac{\partial}{\partial r}\left(\frac{\partial u}{\partial r}+\nu \frac{u}{r}\right)+\frac{\frac{\partial u}{\partial r}+\nu \frac{u}{r}-\left(\frac{u}{r}+\nu \frac{\partial u}{\partial r}\right)}{r} \\
& =\frac{d^{2} u}{d r^{2}}+\frac{\nu}{r} \frac{\partial u}{\partial r}-\nu \frac{u}{r^{2}}+\frac{1}{r} \frac{\partial u}{\partial r}+\nu \frac{u}{r^{2}}-\frac{u}{r^{2}}-\frac{\nu}{r} \frac{\partial u}{\partial r}=\frac{d^{2} u}{d r^{2}}+\frac{1}{r} \frac{\partial u}{\partial r}-\frac{u}{r^{2}} \\
& =\frac{d^{2} u}{d r^{2}}+\frac{\partial}{\partial r} \frac{u}{r}=\frac{\partial}{\partial r}\left(\frac{1}{r}\left(r \frac{\partial u}{\partial r}+u\right)\right)=\frac{\partial}{\partial r}\left(\frac{1}{r} \frac{\partial}{\partial r}(u r)\right) \\
\frac{\partial}{\partial r}(u r) & =C r \\
u r & =C \frac{r^{2}}{2}+C_{2} \\
u(r) & =C_{1} r+\frac{C_{2}}{r}, \quad C_{1} \stackrel{\text { def }}{=} \frac{C}{2} .
\end{aligned}
$$

Insertion in (14) gives

$$
\left\{\begin{array}{l}
\sigma_{\mathrm{r}}=\frac{E}{1-\nu^{2}}\left(\frac{\partial\left(C_{1} r+\frac{C_{2}}{r}\right)}{\partial r}+\nu \frac{\left.C_{1} r+\frac{C_{2}}{r}\right)}{r}\right)=\frac{E}{1-\nu^{2}}\left((1+\nu) C_{1}-\frac{(1-\nu) C_{2}}{r^{2}}\right) \\
\sigma_{\theta}=\frac{E}{1-\nu^{2}}\left(\frac{C_{1} r+\frac{C_{2}}{r}}{r}+\nu \frac{\partial\left(C_{1} r+\frac{C_{2}}{r}\right)}{\partial r}\right)=\frac{E}{1-\nu^{2}}\left((1+\nu) C_{1}+\frac{(1-\nu) C_{2}}{r^{2}}\right) .
\end{array}\right.
$$

Recall from Figure 10 that for stresses $\sigma_{x}$, a positive sign means stretching/expansion, whereas for pressures we follow the convention that positive signs means compression. Hence, for a wedge, barrel or rod with an inner radius $r_{\mathrm{i}}=r_{\mathrm{i}}(l)\left(r_{\mathrm{i}}=0\right.$ for the rod) and outer radius $r_{\mathrm{o}}=r_{\mathrm{o}}(l)$, we obtain the boundary conditions

$$
\sigma_{\mathrm{r}}\left(r_{\mathrm{i}}+u\left(r_{\mathrm{i}}, l\right), l\right)=-p_{\mathrm{i}} \quad \text { and } \quad \sigma_{\mathrm{r}}\left(r_{\mathrm{o}}+u\left(r_{\mathrm{o}}, l\right), l\right)=-p_{\mathrm{o}}
$$

for compressive pressures $\left(p_{\mathrm{i}}, p_{\mathrm{o}}>0\right)$ in radial direction at the inner and outer surface, respectively:

$$
\left\{\begin{array} { l } 
{ \frac { E } { 1 - \nu ^ { 2 } } ( ( 1 + \nu ) C _ { 1 } - \frac { ( 1 - \nu ) C _ { 2 } } { r _ { \mathrm { i } } ^ { 2 } } ) = - p _ { \mathrm { i } } } \\
{ \frac { E } { 1 - \nu ^ { 2 } } ( ( 1 + \nu ) C _ { 1 } - \frac { ( 1 - \nu ) C _ { 2 } } { r _ { \mathrm { o } } ^ { 2 } } ) = - p _ { \mathrm { o } } , }
\end{array} \quad \Longleftrightarrow \quad \left\{\begin{array}{l}
C_{1}=\frac{1-\nu}{E} \frac{p_{\mathrm{i}} r_{\mathrm{i}}^{2}-p_{\mathrm{o}} r_{\mathrm{o}}^{2}}{r_{\mathrm{o}}^{2}-r_{\mathrm{i}}^{2}} \\
C_{2}=\frac{1+\nu}{E} \frac{\left(p_{\mathrm{i}}-p_{\mathrm{o}}\right)\left(r_{\mathrm{i}} r_{\mathrm{o}}\right)^{2}}{r_{\mathrm{o}}^{2}-r_{\mathrm{i}}^{2}} .
\end{array}\right.\right.
$$

The only difference for the rod is that $C_{2}=0$ (or we would have infinite displacements in the centre), so that we only have the second of these boundary conditions. For all these cases (with $\left(r_{\mathrm{i}}=0\right)$ for the rod), insertion into (15) gives

$$
u(r, l)=\frac{1-\nu}{E} \frac{p_{\mathrm{i}} r_{\mathrm{i}}^{2}-p_{\mathrm{o}} r_{\mathrm{o}}^{2}}{r_{\mathrm{o}}^{2}-r_{\mathrm{i}}^{2}} r+\frac{1+\nu}{E} \frac{\left(p_{\mathrm{i}}-p_{\mathrm{o}}\right)\left(r_{\mathrm{i}} r_{\mathrm{o}}\right)^{2}}{r_{\mathrm{o}}^{2}-r_{\mathrm{i}}^{2}} \frac{1}{r},
$$

where the entire dependence on $l$ is contained in the boundary conditions via $p_{\mathrm{o}}=p_{\mathrm{o}}(l)$, $p_{\mathrm{i}}=p_{\mathrm{i}}(l), r_{\mathrm{o}}=r_{\mathrm{o}}(l)$ and $r_{\mathrm{i}}=r_{\mathrm{i}}(l)$.

The left-hand side of Figure 12 shows the pressure $P_{1}$ acting on the rod, compressing it from radius $r_{\text {ro }}$ to the radius $n_{1}$ in Figure 2 (page 5). The resulting surface displacement is

$$
u_{\text {ro }} \stackrel{\text { def }}{=} n_{1}-r_{\text {ro }},
$$




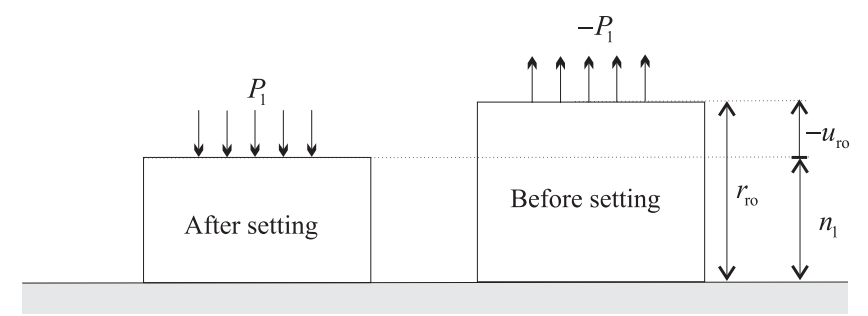

Figure 12: Deformation of the rod during the setting of the wedge.

with positive sign indicating a displacement away from the centre, as in Figure 10 (b).

Now recall that in (17), $r_{\mathrm{i}}(l)$ and $r_{\mathrm{o}}(l)$ are the surface radii before the surface displacement and, from (16), that $p_{\mathrm{i}}$ and $p_{\mathrm{o}}$ are the pressures at radius $r_{\mathrm{i}}+u\left(r_{\mathrm{i}}, l\right)$ and $r_{\mathrm{o}}+u\left(r_{\mathrm{o}}, l\right)$, respectively. Thus, a direct application of (17) to the left-hand half of Figure 12 gives

$$
u_{\mathrm{ro}}=\left.u\left(r_{\mathrm{ro}}\right)\right|_{\nu=\nu_{\mathrm{r}}, E=E_{\mathrm{r}}, p_{\mathrm{o}}=P_{1}, r_{\mathrm{i}}=0, r_{\mathrm{o}}=r_{\mathrm{ro}}}=P_{1} r_{\mathrm{ro}} \frac{1-\nu_{\mathrm{r}}}{E_{\mathrm{r}}} .
$$

In order to rather get equations in the unknown radii $n_{1}, n_{2}$ and $n_{3}$, we will from now on assume all materials to be linearly elastic with the same modulus of elasticity both in compression and tension. Then, instead of the pressure $P_{1}$ compressing the rod outer radius $r_{\text {ro }}$ to outer radius $n_{1}$, it can be equally stated that the pressure $-P_{1}$ would expand a rod of outer radius $n_{1}$ to outer radius $r_{\text {ro }}$, as indicated in the right-hand half of Figure 12 With this interchange of the roles of $r_{\text {ro }}$ and $n_{1}$, (17) gives $u_{\text {ro }}$ in terms of the unknown radius $n_{1}$ :

$$
-u_{\mathrm{ro}}=\left.u\left(n_{1}\right)\right|_{\nu=\nu_{\mathrm{r}}, E=E_{\mathrm{r}}, p_{\mathrm{o}}=-P_{1}, r_{\mathrm{i}}=0, r_{\mathrm{o}}=n_{1}}=P_{1} n_{1} \frac{1-\nu_{\mathrm{r}}}{E_{\mathrm{r}}} .
$$

It remains to repeat the same argument for first the wedge and then the barrel. Figure 13 shows the radii of and pressures acting on the wedge inner and outer surfaces. The displacement of the inner surface is

$$
u_{\mathrm{wi}} \stackrel{\text { def }}{=} n_{1}-r_{\mathrm{wi}} \text {. Note: We will always have } r_{\mathrm{wi}}=r_{\mathrm{ro}} \text {, that is, } u_{\mathrm{wi}}=u_{\mathrm{ro}} \text {. }
$$

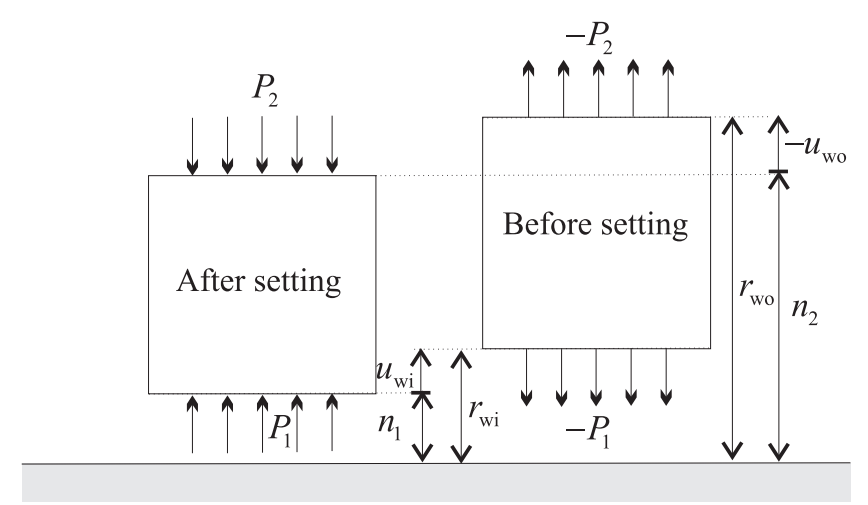

Figure 13: Deformation of the wedge during its setting. 
Hence, (17) gives that

$$
\begin{aligned}
-u_{\mathrm{wi}} & =\left.u\left(n_{1}\right)\right|_{\nu=\nu_{\mathrm{w}}, E=E_{\mathrm{w}}, p_{\mathrm{i}}=-P_{1}, p_{\mathrm{o}}=-P_{2}, r_{\mathrm{i}}=n_{1}, r_{\mathrm{o}}=n_{2}} \\
& =\frac{1-\nu_{\mathrm{w}}}{E_{\mathrm{w}}} \frac{-P_{1} n_{1}^{2}+P_{2} n_{2}^{2}}{n_{2}^{2}-n_{1}^{2}} n_{1}+\frac{1+\nu_{\mathrm{w}}}{E_{\mathrm{w}}} \frac{\left(-P_{1}+P_{2}\right)\left(n_{1} n_{2}\right)^{2}}{n_{2}^{2}-n_{1}^{2}} \frac{1}{n_{1}} \\
& =\frac{n_{1}}{E_{\mathrm{w}}\left(n_{2}^{2}-n_{1}^{2}\right)}\left(\left(1-\nu_{\mathrm{w}}\right)\left(P_{2} n_{2}^{2}-P_{1} n_{1}^{2}\right)+\left(1+\nu_{\mathrm{w}}\right)\left(P_{2}-P_{1}\right) n_{2}^{2}\right) \\
& =\frac{n_{1}}{E_{\mathrm{w}}\left(n_{2}^{2}-n_{1}^{2}\right)}\left(2 P_{2} n_{2}^{2}-P_{1}\left(\left(1-\nu_{\mathrm{w}}\right) n_{1}^{2}+\left(1+\nu_{\mathrm{w}}\right) n_{2}^{2}\right)\right) .
\end{aligned}
$$

Similarly, for the outer surface displacement,

$$
\begin{aligned}
u_{\mathrm{wo}} & \stackrel{\text { def }}{=} n_{2}-r_{\mathrm{wo}} \quad \text { and } \\
-u_{\mathrm{wo}} & =\left.u\left(n_{2}\right)\right|_{\nu=\nu_{\mathrm{w}}, E=E_{\mathrm{w}}, p_{\mathrm{i}}=-P_{1}, p_{\mathrm{o}}=-P_{2}, r_{\mathrm{i}}=n_{1}, r_{\mathrm{o}}=n_{2}} \\
& =\frac{1-\nu_{\mathrm{w}}-P_{1} n_{1}^{2}+P_{2} n_{2}^{2}}{E_{\mathrm{w}}} n_{2}+\frac{1+\nu_{\mathrm{w}}}{E_{\mathrm{w}}} \frac{\left(-P_{1}+P_{2}\right)\left(n_{1} n_{2}\right)^{2}}{n_{2}^{2}-n_{1}^{2}} \frac{1}{n_{2}} \\
& =\frac{n_{2}}{E_{\mathrm{w}}\left(n_{2}^{2}-n_{1}^{2}\right)}\left(\left(1-\nu_{\mathrm{w}}\right)\left(P_{2} n_{2}^{2}-P_{1} n_{1}^{2}\right)+\left(1+\nu_{\mathrm{w}}\right)\left(P_{2}-P_{1}\right) n_{1}^{2}\right) \\
& =\frac{n_{2}}{E_{\mathrm{w}}\left(n_{2}^{2}-n_{1}^{2}\right)}\left(P_{2}\left(\left(1-\nu_{\mathrm{w}}\right) n_{2}^{2}+\left(1+\nu_{\mathrm{w}}\right) n_{1}^{2}\right)-2 P_{1} n_{1}^{2}\right) .
\end{aligned}
$$

For the barrel inner surface in Figure 14,

$$
\begin{aligned}
u_{\mathrm{bi}} & \stackrel{\text { def }}{=} n_{2}-r_{\mathrm{bi}} \quad \text { and } \\
-u_{\mathrm{bi}} & =\left.u\left(n_{2}\right)\right|_{\nu=\nu_{\mathrm{b}}, E=E_{\mathrm{b}}, p_{\mathrm{i}}=-P_{2}, p_{\mathrm{o}}=0, r_{\mathrm{i}}=n_{2}, r_{\mathrm{o}}=n_{3}} \\
& =\frac{1-\nu_{\mathrm{b}}}{E_{\mathrm{b}}} \frac{-P_{2} n_{2}^{2}}{n_{3}^{2}-n_{2}^{2}} n_{2}+\frac{1+\nu_{\mathrm{b}}}{E_{\mathrm{b}}} \frac{-P_{2}\left(n_{2} n_{3}\right)^{2}}{n_{3}^{2}-n_{2}^{2}} \frac{1}{n_{2}} \\
& =-\frac{P_{2} n_{2}}{E_{\mathrm{b}}\left(n_{3}^{2}-n_{2}^{2}\right)}\left(\left(1-\nu_{\mathrm{b}}\right) n_{2}^{2}+\left(1+\nu_{\mathrm{b}}\right) n_{3}^{2}\right) .
\end{aligned}
$$

In the same way for the barrel outer surface,

$$
\begin{aligned}
u_{\mathrm{bo}} \stackrel{\text { def }}{=} n_{3}-r_{\mathrm{bo}}-u_{\mathrm{bo}} & =\left.u\left(n_{3}\right)\right|_{\nu=\nu_{\mathrm{b}}, E=E_{\mathrm{b}}, p_{\mathrm{i}}=-P_{2}, p_{\mathrm{o}}=0, r_{\mathrm{i}}=n_{2}, r_{\mathrm{o}}=n_{3}} \\
& =\frac{1-\nu_{\mathrm{b}}}{E_{\mathrm{b}}} \frac{-P_{2} n_{2}^{2}}{n_{3}^{2}-n_{2}^{2}} n_{3}+\frac{1+\nu_{\mathrm{b}}}{E_{\mathrm{b}}} \frac{-P_{2}\left(n_{2} n_{3}\right)^{2}}{n_{3}^{2}-n_{2}^{2}} \frac{1}{n_{3}} \\
& =-\frac{P_{2} n_{2}^{2} n_{3}}{E_{\mathrm{b}}\left(n_{3}^{2}-n_{2}^{2}\right)}\left(1-\nu_{\mathrm{b}}+1+\nu_{\mathrm{b}}\right)=-\frac{2 P_{2} n_{2}^{2} n_{3}}{E_{\mathrm{b}}\left(n_{3}^{2}-n_{2}^{2}\right)} .
\end{aligned}
$$

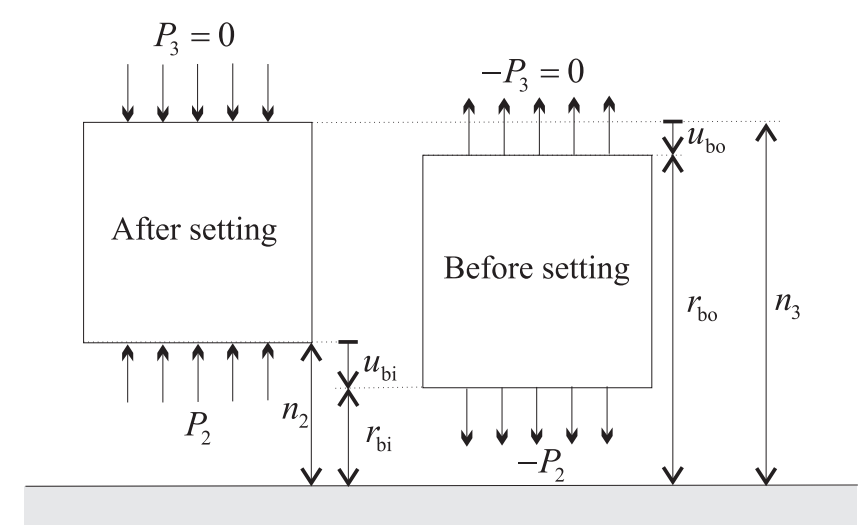

Figure 14: Deformation of the barrel during the setting of the wedge. 
We have now derived a system of 10 equations (18) in 10 unknowns, which, after pairwise combination of equations with the same left-hand side, can be rearranged as follows:

$$
\begin{array}{rlr}
r_{\mathrm{ro}}-n_{1}=P_{1} n_{1} \frac{1-\nu_{\mathrm{r}}}{E_{\mathrm{r}}} & P_{1}, n_{1} \\
r_{\mathrm{wi}}-n_{1}=\frac{n_{1}}{E_{\mathrm{w}}\left(n_{2}^{2}-n_{1}^{2}\right)}\left(2 P_{2} n_{2}^{2}-P_{1}\left(\left(1-\nu_{\mathrm{w}}\right) n_{1}^{2}+\left(1+\nu_{\mathrm{w}}\right) n_{2}^{2}\right)\right) & P_{1}, P_{2}, n_{1}, n_{2} \\
r_{\mathrm{wo}}-n_{2}=\frac{n_{2}}{E_{\mathrm{w}}\left(n_{2}^{2}-n_{1}^{2}\right)}\left(P_{2}\left(\left(1-\nu_{\mathrm{w}}\right) n_{2}^{2}+\left(1+\nu_{\mathrm{w}}\right) n_{1}^{2}\right)-2 P_{1} n_{1}^{2}\right) & P_{1}, P_{2}, n_{1}, n_{2} \\
r_{\mathrm{bi}}-n_{2}=-\frac{P_{2} n_{2}}{E_{\mathrm{b}}\left(n_{3}^{2}-n_{2}^{2}\right)}\left(\left(1-\nu_{\mathrm{b}}\right) n_{2}^{2}+\left(1+\nu_{\mathrm{b}}\right) n_{3}^{2}\right) & P_{2}, n_{2}, n_{3} \\
r_{\mathrm{bo}}-n_{3}=-\frac{2 P_{2} n_{2}^{2} n_{3}}{E_{\mathrm{b}}\left(n_{3}^{2}-n_{2}^{2}\right)} & P_{2}, n_{2}, n_{3} \\
u_{\mathrm{ro}} \stackrel{\text { def }}{=} n_{1}-r_{\mathrm{ro}} & u_{\mathrm{ro}}, n_{1} \\
u_{\mathrm{wi}} \stackrel{\text { def }}{=} n_{1}-r_{\mathrm{wi}} & u_{\mathrm{wi}}, n_{1} \\
u_{\mathrm{wo}} \stackrel{\text { def }}{=} n_{2}-r_{\mathrm{wo}} & u_{\mathrm{wo}}, n_{2} \\
u_{\mathrm{bi}} \stackrel{\text { def }}{=} n_{2}-r_{\mathrm{bi}} & u_{\mathrm{bi}}, n_{2} \\
u_{\mathrm{bo}} \stackrel{\text { def }}{=} n_{3}-r_{\mathrm{bo}} & u_{\mathrm{bo}}, n_{3}
\end{array}
$$

Hence, only the five equations (19a)-(19e) in five unknowns have to be solved, and the equations (19f) - 19j] then immediately give the corresponding displacements.

Remark 3. The plane stress assumption is of main interest for us, but the fact that $\varepsilon_{\mathrm{r}}$ and $\varepsilon_{\theta}$ get an almost identical form under a plane strain assumption is interesting. In fact, we then have $\varepsilon_{1}=0$, which upon insertion into the equation for $\varepsilon_{1}$ gives $\sigma_{1}=\nu\left(\sigma_{\mathrm{r}}+\sigma_{\theta}\right)$, so that the equations for $\varepsilon_{\mathrm{r}}$ and $\varepsilon_{\theta}$ become

$$
\begin{gathered}
\varepsilon_{\mathrm{r}}=\frac{\sigma_{\mathrm{r}}-\nu\left(\sigma_{\theta}+\nu\left(\sigma_{\mathrm{r}}+\sigma_{\theta}\right)\right)}{E}=\frac{\left(1-\nu^{2}\right)\left(\sigma_{\mathrm{r}}-\nu \sigma_{\theta} \frac{1+\nu}{1-\nu^{2}}\right)}{E} \\
\varepsilon_{\theta}=\frac{\sigma_{\theta}-\nu\left(\sigma_{\mathrm{r}}+\nu\left(\sigma_{\mathrm{r}}+\sigma_{\theta}\right)\right)}{E}=\frac{\left(1-\nu^{2}\right)\left(\sigma_{\theta}-\nu \sigma_{\mathrm{r}} \frac{1+\nu}{1-\nu^{2}}\right)}{E} .
\end{gathered}
$$

Hence the plane strain correspondence to (13) is

$$
\varepsilon_{\mathrm{r}}=\frac{\sigma_{\mathrm{r}}-\nu^{*} \sigma_{\theta}}{E^{*}}, \quad \varepsilon_{\theta}=\frac{\sigma_{\theta}-\nu^{*} \sigma_{\mathrm{r}}}{E^{*}} \quad \text { and } \quad \varepsilon_{1}=0
$$

with $E^{*}=\frac{E}{1-\nu^{2}}$ and $\nu^{*}=\frac{\nu}{1-\nu}$. 


\section{B Proofs of propositions $1-3$}

Proof of Proposition 1. If $\frac{P_{2}}{P_{1}} \neq \frac{E_{\mathrm{w}}\left(1-\nu_{\mathrm{r}}\right)+E_{\mathrm{r}}\left(1+\nu_{\mathrm{w}}\right)}{2 E_{\mathrm{r}}}$, then it follows from (2b) that

$$
\begin{aligned}
\frac{n_{2}\left(P_{1}, P_{2}\right)}{n_{1}\left(P_{1}\right)} & =\sqrt{\frac{\frac{E_{\mathrm{w}} r_{\mathrm{wi}}}{n_{1}\left(P_{1}\right)}-E_{\mathrm{w}}-P_{1}\left(1-\nu_{\mathrm{w}}\right)}{\frac{E_{\mathrm{w}} r_{\mathrm{wi}}}{n_{1}\left(P_{1}\right)}-E_{\mathrm{w}}+P_{1}\left(1+\nu_{\mathrm{w}}\right)-2 P_{2}}} \quad(\operatorname{apply}(2 \mathrm{a})) \\
& =\sqrt{\frac{\frac{E_{\mathrm{r}}+P_{1}\left(1-\nu_{\mathrm{r}}\right)}{E_{\mathrm{r}} r_{\mathrm{ro}}} E_{\mathrm{w}} r_{\mathrm{wi}}-E_{\mathrm{w}}-P_{1}\left(1-\nu_{\mathrm{w}}\right)}{\frac{E_{\mathrm{r}}+P_{1}\left(1-\nu_{\mathrm{r}}\right)}{E_{\mathrm{r}} r_{\mathrm{ro}}} E_{\mathrm{w}} r_{\mathrm{wi}}-E_{\mathrm{w}}+P_{1}\left(1+\nu_{\mathrm{w}}\right)-2 P_{2}}} \\
& =\sqrt{\frac{E_{\mathrm{r}} E_{\mathrm{w}} r_{\mathrm{wi}}+E_{\mathrm{w}} r_{\mathrm{wi}}\left(1-\nu_{\mathrm{r}}\right) P_{1}-E_{\mathrm{r}} E_{\mathrm{w}} r_{\mathrm{ro}}-E_{\mathrm{r}} r_{\mathrm{ro}}\left(1-\nu_{\mathrm{w}}\right) P_{1}}{E_{\mathrm{r}} E_{\mathrm{w}} r_{\mathrm{wi}}+E_{\mathrm{w}} r_{\mathrm{wi}}\left(1-\nu_{\mathrm{r}}\right) P_{1}-E_{\mathrm{r}} E_{\mathrm{w}} r_{\mathrm{ro}}+E_{\mathrm{r}} r_{\mathrm{ro}}\left(1+\nu_{\mathrm{w}}\right) P_{1}-2 E_{\mathrm{r}} r_{\mathrm{ro}} P_{2}}}
\end{aligned}
$$

$(\operatorname{apply}(3 \mathrm{C}))=\sqrt{\frac{r_{\mathrm{wi}}\left(E_{\mathrm{w}}\left(1-\nu_{\mathrm{r}}\right)-E_{\mathrm{r}}\left(1-\nu_{\mathrm{w}}\right)\right) P_{1}}{r_{\mathrm{wi}}\left[\left(E_{\mathrm{w}}\left(1-\nu_{\mathrm{r}}\right)+E_{\mathrm{r}}\left(1+\nu_{\mathrm{w}}\right)\right) P_{1}-2 E_{\mathrm{r}} P_{2}\right]}}=\sqrt{\frac{\frac{E_{\mathrm{w}}\left(1-\nu_{\mathrm{r}}\right)-E_{\mathrm{r}}\left(1-\nu_{\mathrm{w}}\right)}{E_{\mathrm{w}}\left(1-\nu_{\mathrm{r}}\right)+E_{\mathrm{r}}\left(1+\nu_{\mathrm{w}}\right)}}{1-\frac{2 E_{\mathrm{r}} P_{2} / P_{1}}{E_{\mathrm{w}}\left(1-\nu_{\mathrm{r}}\right)+E_{\mathrm{r}}\left(1+\nu_{\mathrm{w}}\right)}}}$

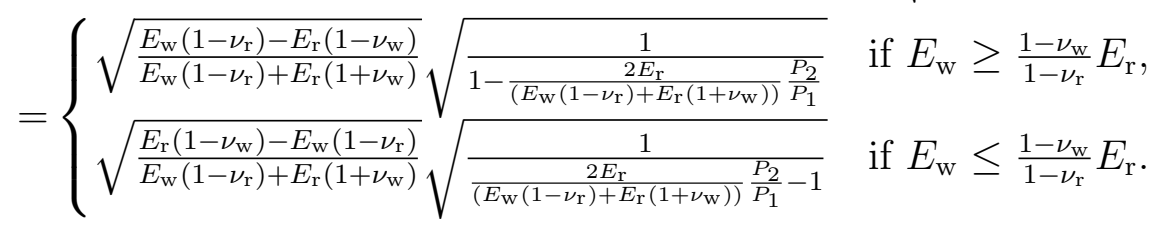

The right-hand denominator is positive if and only if conditions (5) hold and then (6) follows.

The overlapping case $E_{\mathrm{w}}=\frac{1-\nu_{\mathrm{w}}}{1-\nu_{\mathrm{r}}} E_{\mathrm{r}}$ might at first sight seem to give $n_{2}=0$, which violates (3b). Thus, this case can occur only in the sense that the numerator and denominator in (20) converge to zero simultaneously. Every possible choice of parameters in the system of equations (1) gives a "rule" that associates each $E_{w}$ with one particular $P_{2} / P_{1}$, thus governing the speeds of convergence and enabling convergence of $\frac{n_{2}\left(P_{1}, P_{2}\right)}{n_{1}\left(P_{1}\right)}$ to any positive real number. In fact, for $|\varepsilon|<1$ and an arbitrary positive real number $R$, set $\varepsilon_{2} \stackrel{\text { def }}{=} R^{2} \varepsilon$,

$$
\frac{P_{2}}{P_{1}}=\frac{E_{\mathrm{w}}\left(1-\nu_{\mathrm{r}}\right)+E_{\mathrm{r}}\left(1+\nu_{\mathrm{w}}\right)}{2 E_{\mathrm{r}}}(1-\varepsilon) \quad \text { and } \quad E_{\mathrm{w}}=\frac{1-\nu_{\mathrm{w}}}{1-\nu_{\mathrm{r}}} E_{\mathrm{r}}\left(1+\varepsilon_{2}\right) .
$$

Then insertion in (20) gives

$$
\begin{aligned}
& \frac{n_{2}\left(P_{1}, P_{2}\right)}{n_{1}\left(P_{1}\right)}=\sqrt{\frac{\frac{1-\nu_{\mathrm{w}}}{1-\nu_{\mathrm{r}}} E_{\mathrm{r}} \varepsilon_{2}}{\frac{\frac{1-\nu_{\mathrm{w}}}{1-\nu_{\mathrm{r}}} E_{\mathrm{r}}\left(1+\varepsilon_{2}\right)\left(1-\nu_{\mathrm{r}}\right)+E_{\mathrm{r}}\left(1+\nu_{\mathrm{w}}\right)}{\varepsilon}}}=R \sqrt{\frac{\frac{1-\nu_{\mathrm{w}}}{1-\nu_{\mathrm{r}}} E_{\mathrm{r}}}{\frac{1-\nu_{\mathrm{w}}}{1-\nu_{\mathrm{r}}} E_{\mathrm{r}}\left(1+R^{2} \varepsilon\right)\left(1-\nu_{\mathrm{r}}\right)+E_{\mathrm{r}}\left(1+\nu_{\mathrm{w}}\right)}} \\
& \rightarrow R \sqrt{\frac{\frac{1-\nu_{\mathrm{w}}}{1-\nu_{\mathrm{r}}}}{\frac{1-\nu_{\mathrm{w}}}{1-\nu_{\mathrm{r}}}\left(1-\nu_{\mathrm{r}}\right)+1+\nu_{\mathrm{w}}}}=R \sqrt{\frac{1}{2} \frac{1-\nu_{\mathrm{w}}}{1-\nu_{\mathrm{r}}}} \quad \text { as } \varepsilon \rightarrow 0 .
\end{aligned}
$$

Proof of Proposition Q. From (2C) we have that $n_{3}\left(P_{1}, P_{2}\right)=\sqrt{\frac{u_{3}\left(P_{1}, P_{2}\right)}{v_{3}\left(P_{1}, P_{2}\right)}} n_{2}\left(P_{1}, P_{2}\right)$ where $v_{3}\left(P_{1}, P_{2}\right)>$ 0 if and only if

$$
E_{\mathrm{b}} r_{\mathrm{bi}}>\left(E_{\mathrm{b}}-P_{2}\left(1+\nu_{\mathrm{b}}\right)\right) n_{2}\left(P_{1}, P_{2}\right)
$$

Since $E_{\mathrm{b}} r_{\mathrm{bi}}>0$ and, by (5),$n_{2}\left(P_{1}, P_{2}\right)>0$, we have the equivalence

$v_{3}\left(P_{1}, P_{2}\right)>0 \quad \Longleftrightarrow \quad P_{2} \geq \frac{E_{\mathrm{b}}}{1+\nu_{\mathrm{b}}} \quad$ or $\quad\left(P_{2}<\frac{E_{\mathrm{b}}}{1+\nu_{\mathrm{b}}}\right.$ and $\left.n_{2}\left(P_{1}, P_{2}\right)<\frac{E_{\mathrm{b}} r_{\mathrm{bi}}}{E_{\mathrm{b}}-P_{2}\left(1+\nu_{\mathrm{b}}\right)}\right)$. 
Similarly, $u_{3}\left(P_{1}, P_{2}\right)>0$ if and only if $E_{\mathrm{b}} r_{\mathrm{bi}}-\left(E_{\mathrm{b}}+P_{2}\left(1-\nu_{\mathrm{b}}\right)\right) n_{2}\left(P_{1}, P_{2}\right)>0$, or equivalently

$$
u_{3}\left(P_{1}, P_{2}\right)>0 \quad \Longleftrightarrow \quad n_{2}\left(P_{1}, P_{2}\right)<\frac{E_{\mathrm{b}} r_{\mathrm{bi}}}{E_{\mathrm{b}}+P_{2}\left(1-\nu_{\mathrm{b}}\right)} .
$$

Thus we have two different cases

1. Suppose that $P_{2} \geq \frac{E_{\mathrm{b}}}{1+\nu_{\mathrm{b}}}$. Then by (21),$n_{3}\left(P_{1}, P_{2}\right)$ is well-defined by (2c) if and ony if

$$
n_{2}\left(P_{1}, P_{2}\right)<\frac{E_{\mathrm{b}} r_{\mathrm{bi}}}{E_{\mathrm{b}}+P_{2}\left(1-\nu_{\mathrm{b}}\right)} \leq r_{\mathrm{bi}}
$$

which is not possible, due to the constraint $r_{\mathrm{bi}} \leq n_{2}\left(P_{1}, P_{2}\right)$ in (3d).

2. Suppose that $P_{2}<\frac{E_{\mathrm{b}}}{1+\nu_{\mathrm{b}}}$. Then by (21),$n_{3}\left(P_{1}, P_{2}\right)$ is well-defined by (2C) either if both $u_{3}$ and $v_{3}$ are positive or if both are negative

(a) Both $u_{3}$ and $v_{3}$ are positive if

$$
n_{2}\left(P_{1}, P_{2}\right)<\frac{E_{\mathrm{b}} r_{\mathrm{bi}}}{E_{\mathrm{b}}+P_{2}\left(1-\nu_{\mathrm{b}}\right)}\left(\leq r_{\mathrm{bi}} \leq \frac{E_{\mathrm{b}} r_{\mathrm{bi}}}{E_{\mathrm{b}}-P_{2}\left(1+\nu_{\mathrm{b}}\right)}\right)
$$

which is not possible, due to the constraint $r_{\mathrm{bi}} \leq n_{2}\left(P_{1}, P_{2}\right)$ in (3d).

(b) Both $u_{3}$ and $v_{3}$ are negative if

$$
n_{2}\left(P_{1}, P_{2}\right)>\frac{E_{\mathrm{b}} r_{\mathrm{bi}}}{E_{\mathrm{b}}-P_{2}\left(1+\nu_{\mathrm{b}}\right)}\left(\geq r_{\mathrm{bi}} \geq \frac{E_{\mathrm{b}} r_{\mathrm{bi}}}{E_{\mathrm{b}}+P_{2}\left(1-\nu_{\mathrm{b}}\right)}\right),
$$

which therefore is a sharpening of the constraint $r_{\mathrm{bi}} \leq n_{2}\left(P_{1}, P_{2}\right)$ in (3d).

This gives exactly the sharpened constraint (17).

Proof of Proposition 3. We can reformulate both the upper and lower bounds on $n_{2}$ in (7) in the following way (or with $<$ replaced by $\geq$ ), with a positive bound $M\left(P_{2}\right)$ :

$$
\begin{aligned}
& M\left(P_{2}\right)<n_{2}\left(P_{1}, P_{2}\right) \\
& M\left(P_{2}\right)^{2}<\frac{E_{\mathrm{w}} r_{\mathrm{wi}} n_{1}\left(P_{1}\right)^{2}-\left(E_{\mathrm{w}}+P_{1}\left(1-\nu_{\mathrm{w}}\right)\right) n_{1}\left(P_{1}\right)^{3}}{E_{\mathrm{w}} r_{\mathrm{wi}}+\left(P_{1}\left(1+\nu_{\mathrm{w}}\right)-2 P_{2}-E_{\mathrm{w}}\right) n_{1}\left(P_{1}\right)} \\
& \frac{M\left(P_{2}\right)^{2}}{n_{1}\left(P_{1}\right)^{2}}<\frac{\frac{E_{\mathrm{w}} r_{\mathrm{wi}}}{n_{1}\left(P_{1}\right)}-E_{\mathrm{w}}-P_{1}\left(1-\nu_{\mathrm{w}}\right)}{\frac{E_{\mathrm{w}} r_{\mathrm{wi}}}{n_{1}\left(P_{1}\right)}+P_{1}\left(1+\nu_{\mathrm{w}}\right)-2 P_{2}-E_{\mathrm{w}}} \quad(\text { apply (2a) and }(\underline{3 \mathrm{c}})) \\
& \frac{M\left(P_{2}\right)^{2}\left(E_{\mathrm{r}}+P_{1}\left(1-\nu_{\mathrm{r}}\right)\right)^{2}}{E_{\mathrm{r}}^{2} r_{\mathrm{ro}}^{2}}<\frac{\frac{E_{\mathrm{w}} r_{\mathrm{ro}}\left(E_{\mathrm{r}}+P_{1}\left(1-\nu_{\mathrm{r}}\right)\right)}{E_{\mathrm{r}} r_{\mathrm{ro}}}-E_{\mathrm{w}}-P_{1}\left(1-\nu_{\mathrm{w}}\right)}{\frac{E_{\mathrm{w}} r_{\mathrm{ro}}\left(E_{\mathrm{r}}+P_{\mathrm{r}}\left(1-\nu_{\mathrm{r}}\right)\right)}{E_{\mathrm{r}} r_{\mathrm{ro}}}+P_{1}\left(1+\nu_{\mathrm{w}}\right)-2 P_{2}-E_{\mathrm{w}}} \\
& =\frac{\frac{E_{\mathrm{w}}\left(1-\nu_{\mathrm{r}}\right)-E_{\mathrm{r}}\left(1-\nu_{\mathrm{w}}\right)}{E_{\mathrm{r}}} P_{1}}{\frac{E_{\mathrm{w}}\left(1-\nu_{\mathrm{r}}\right)+E_{\mathrm{r}}\left(1+\nu_{\mathrm{w}}\right)}{E_{\mathrm{r}}} P_{1}-2 P_{2}}
\end{aligned}
$$

We know from Proposition 1 that the numerator and denominator in the right-hand side fraction are either both positive, both negative or both vanishing. This gives three different cases:

1. If $E_{\mathrm{w}}>E_{\mathrm{r}} \frac{1-\nu_{\mathrm{w}}}{1-\nu_{\mathrm{r}}}$, then the numerator and the denominator in (23) are both positive. Hence, the constraint (77) consists of the constraint $0<P_{2}<\frac{E_{\mathrm{b}}}{1+\nu_{\mathrm{b}}}$ and a double inequality that can be reformulated into the following two constraints: 
(a) By setting $M\left(P_{2}\right)=\frac{E_{\mathrm{b}} r_{\mathrm{bi}}}{E_{\mathrm{b}}-P_{2}\left(1+\nu_{\mathrm{b}}\right)}$ in (22), we get

$$
\begin{aligned}
\frac{E_{\mathrm{b}}^{2} r_{\mathrm{bi}}^{2}\left(E_{\mathrm{r}}+P_{1}\left(1-\nu_{\mathrm{r}}\right)\right)^{2}}{E_{\mathrm{r}}^{2} r_{\mathrm{ro}}^{2}\left(E_{\mathrm{b}}-P_{2}\left(1+\nu_{\mathrm{b}}\right)\right)^{2}}\left(\frac{E_{\mathrm{w}}\left(1-\nu_{\mathrm{r}}\right)+E_{\mathrm{r}}\left(1+\nu_{\mathrm{w}}\right)}{E_{\mathrm{r}}} P_{1}-2 P_{2}\right) & <\frac{E_{\mathrm{w}}\left(1-\nu_{\mathrm{r}}\right)-E_{\mathrm{r}}\left(1-\nu_{\mathrm{w}}\right)}{E_{\mathrm{r}}} P_{1} \\
\frac{E_{\mathrm{w}}\left(1-\nu_{\mathrm{r}}\right)+E_{\mathrm{r}}\left(1+\nu_{\mathrm{w}}\right)}{E_{\mathrm{r}}} P_{1}-2 P_{2} & <\frac{E_{\mathrm{w}}\left(1-\nu_{\mathrm{r}}\right)-E_{\mathrm{r}}\left(1-\nu_{\mathrm{w}}\right)}{E_{\mathrm{r}}} P_{1} \frac{E_{\mathrm{r}}^{2} r_{\mathrm{ro}}^{2}\left(E_{\mathrm{b}}-P_{2}\left(1+\nu_{\mathrm{b}}\right)\right)^{2}}{E_{\mathrm{b}}^{2} r_{\mathrm{bi}}^{2}\left(E_{\mathrm{r}}+P_{1}\left(1-\nu_{\mathrm{r}}\right)\right)^{2}} \\
(\operatorname{apply}(8)) & \frac{E_{\mathrm{w}}\left(1-\nu_{\mathrm{r}}\right)+E_{\mathrm{r}}\left(1+\nu_{\mathrm{w}}\right)}{E_{\mathrm{r}}} P_{1}-2 P_{2}<h\left(P_{1}\right)\left(E_{\mathrm{b}}^{2}-2 E_{\mathrm{b}}\left(1+\nu_{\mathrm{b}}\right) P_{2}+\left(1+\nu_{\mathrm{b}}\right)^{2} P_{2}^{2}\right) .
\end{aligned}
$$

Since $E_{\mathrm{w}}>E_{\mathrm{r}} \frac{1-\nu_{\mathrm{w}}}{1-\nu_{\mathrm{r}}}$, it follows from (8) that $h\left(P_{1}\right)>0$, so that

$$
\begin{aligned}
P_{2}^{2}+2 \frac{1-h\left(P_{1}\right) E_{\mathrm{b}}\left(1+\nu_{\mathrm{b}}\right)}{h\left(P_{1}\right)\left(1+\nu_{\mathrm{b}}\right)^{2}} & P_{2}+\frac{h\left(P_{1}\right) E_{\mathrm{b}}^{2}-\frac{E_{\mathrm{w}}\left(1-\nu_{\mathrm{r}}\right)+E_{\mathrm{r}}\left(1+\nu_{\mathrm{w}}\right)}{E_{\mathrm{r}}} P_{1}}{h\left(P_{1}\right)\left(1+\nu_{\mathrm{b}}\right)^{2}}>0 \\
& \mathcal{P}_{P_{1}}\left(P_{2}\right) \stackrel{\text { def }}{=} P_{2}^{2}+2 a\left(P_{1}\right) P_{2}+b\left(P_{1}\right)>0 .
\end{aligned}
$$

The polynomial $\mathcal{P}_{P_{1}}$ has (complex- or real-valued) roots $-a\left(P_{1}\right) \pm \sqrt{a\left(P_{1}\right)^{2}-b\left(P_{1}\right)}$. Since $\mathcal{P}_{P_{1}}\left(P_{2}\right) \rightarrow \infty$ when $\left|P_{2}\right| \rightarrow \infty$, the minimum of $\mathcal{P}_{P_{1}}$ is $\mathcal{P}_{P_{1}}\left(-a\left(P_{1}\right)\right)$. Thus we get two possible solution sets depending on the sign of $\mathcal{P}_{P_{1}}\left(-a\left(P_{1}\right)\right)$ :

i. If $\mathcal{P}_{P_{1}}\left(-a\left(P_{1}\right)\right) \leq 0$, then $\mathcal{P}_{P_{1}}\left(P_{2}\right)>0$ only for all $P_{2}<-a\left(P_{1}\right)-\sqrt{a\left(P_{1}\right)^{2}-b\left(P_{1}\right)}$ and for all $P_{2}>-a\left(P_{1}\right)+\sqrt{a\left(P_{1}\right)^{2}-b\left(P_{1}\right)}$.

ii. If $\mathcal{P}_{P_{1}}\left(-a\left(P_{1}\right)\right)>0$, then $\mathcal{P}_{P_{1}}\left(P_{2}\right)>0$ for all $P_{2}$.

This gives (9b).

(b) For $M\left(P_{2}\right)=r_{\text {wo }}$ and with $<$ replaced by $\geq$ in (22), (23) becomes

$$
\begin{aligned}
\frac{r_{\mathrm{wo}}^{2}\left(E_{\mathrm{r}}+P_{1}\left(1-\nu_{\mathrm{r}}\right)\right)^{2}}{E_{\mathrm{r}}^{2} r_{\mathrm{ro}}^{2}}\left(\frac{E_{\mathrm{w}}\left(1-\nu_{\mathrm{r}}\right)+E_{\mathrm{r}}\left(1+\nu_{\mathrm{w}}\right)}{E_{\mathrm{r}}} P_{1}-2 P_{2}\right) & \geq \frac{E_{\mathrm{w}}\left(1-\nu_{\mathrm{r}}\right)-E_{\mathrm{r}}\left(1-\nu_{\mathrm{w}}\right)}{E_{\mathrm{r}}} P_{1} \\
\frac{E_{\mathrm{w}}\left(1-\nu_{\mathrm{r}}\right)+E_{\mathrm{r}}\left(1+\nu_{\mathrm{w}}\right)}{E_{\mathrm{r}}} & P_{1}-2 P_{2} \geq \frac{E_{\mathrm{w}}\left(1-\nu_{\mathrm{r}}\right)-E_{\mathrm{r}}\left(1-\nu_{\mathrm{w}}\right)}{E_{\mathrm{r}}} \frac{E_{\mathrm{r}}^{2} r_{\mathrm{ro}}^{2}}{\left(E_{\mathrm{r}}+P_{1}\left(1-\nu_{\mathrm{r}}\right)\right)^{2} r_{\mathrm{wo}}^{2}} P_{1}
\end{aligned}
$$

which gives a sharpening of (5a) to

$$
P_{2} \leq \frac{1}{2}\left(\frac{E_{\mathrm{w}}\left(1-\nu_{\mathrm{r}}\right)+E_{\mathrm{r}}\left(1+\nu_{\mathrm{w}}\right)}{E_{\mathrm{r}}}-\frac{E_{\mathrm{w}}\left(1-\nu_{\mathrm{r}}\right)-E_{\mathrm{r}}\left(1-\nu_{\mathrm{w}}\right)}{\left(E_{\mathrm{r}}+P_{1}\left(1-\nu_{\mathrm{r}}\right)\right)^{2}} E_{\mathrm{r}} \frac{r_{\mathrm{ro}}^{2}}{r_{\mathrm{wo}}^{2}}\right) P_{1},
$$

From this and (77) we get the constraint (9a).

2. Suppose that $E_{\mathrm{w}}<E_{\mathrm{r}} \frac{1-\nu_{\mathrm{w}}}{1-\nu_{\mathrm{r}}}$. The only differences from 1 are that we now start off with negative numerator and denominator in (23), replacing $<$ with $>$ in (24) and then, since also $h\left(P_{1}\right)$ is negative, change from $<$ back to $>$ in (25), thus leaving the constraint (9b) unchanged. Finally, $\geq$ gets replaced by $\leq$ in (26), thus giving a sharpening of (5b) to

$$
P_{2} \geq \frac{1}{2}\left(\frac{E_{\mathrm{w}}\left(1-\nu_{\mathrm{r}}\right)+E_{\mathrm{r}}\left(1+\nu_{\mathrm{w}}\right)}{E_{\mathrm{r}}}-\frac{E_{\mathrm{w}}\left(1-\nu_{\mathrm{r}}\right)-E_{\mathrm{r}}\left(1-\nu_{\mathrm{w}}\right)}{\left(E_{\mathrm{r}}+P_{1}\left(1-\nu_{\mathrm{r}}\right)\right)^{2}} E_{\mathrm{r}} \frac{r_{\mathrm{ro}}^{2}}{r_{\mathrm{wo}}^{2}}\right) P_{1},
$$

From this and (17) we get the constraint (10).

3. If $E_{\mathrm{w}}=E_{\mathrm{r}} \frac{1-\nu_{\mathrm{w}}}{1-\nu_{\mathrm{r}}}$ then recall from Proposition 1 1 that $\frac{P_{2}}{P_{1}}=\frac{1}{2} \frac{E_{\mathrm{w}}\left(1-\nu_{\mathrm{r}}\right)+E_{\mathrm{r}}\left(1+\nu_{\mathrm{w}}\right)}{E_{\mathrm{r}}}$. 


\section{References}

[AMSP01a] A. Al-Mayah, K. A. Soudki, and A. Plumtree. Experimental and analytical investigation of a stainless steel anchorage for CFRP prestressing tendons. PCI Journal, pages 88-100, March-April 2001. 2, 13

[AMSP01b] A. Al-Mayah, K. A. Soudki, and A. Plumtree. Mechanical behavior of CFRP rod anchors under tensile loading. J. of Compos. for Constr., pages 128-135, May 2001. 2

[AMSP06] A. Al-Mayah, K. A. Soudki, and A. Plumtree. Development and assessment of a new CFRP rod-anchor system for prestressed concrete. Appl. Compos. Mater., 13:321-334, 2006. 2

[AMSP07] A. Al-Mayah, K. A. Soudki, and A. Plumtree. Novel anchor system for CFRP rod: Finite-element and mathematical models. J. of Compos. for Constr., pages 469-476, Sept./Oct. 2007. 2, 4, 13,

[BC95] A. H. Burr and J. B. Cheatham. Mechanical analysis and design. Prentice Hall, Englewood Cliffs, NJ, USA, 1995. 3

[BGS10] Anders Bennitz, Niklas Grip, and Jacob W. Schmidt. Thick-walled cylinder theory applied on a conical wedge anchorage — research report with MATLAB implementation documentation. Research report, 2010. WWW: http://pure.ltu.se/ws/fbspretrieve/5135641, 10

[CM91] M. P. Collins and D. Mitchell. Prestressed Concrete Structures. Prentice Hall, Englewood Cliffs, NJ, USA, 1991. 2

[LB82] T. Y. Lin and N. H. Burns. Design of Prestressed Concrete Structures. John Wiley \& Sons, NY, USA, third edition edition, 1982. 2

[Lun00] H. Lundh. Grundläggande hållfasthetslära. Kungliga tekniska högskolan, Stockholm, Sweden, 2000. 3

[SAS98] E. Y. Sayed-Ahmed and N. G. Shrive. A new steel anchorage system for posttensioning applications using carbon fibre reinforced plastic tendons. Can. J. Civ. Eng., 25(113-127), 1998. 2

[SS06] E. Shaheen and N. G. Shrive. Reactive powder concrete anchorage for posttensioning with carbon fiber-reinforced polymer tendons. ACI Mater. J., pages 436-443, Nov-Dec 2006. 4

[Wan53] C. T. Wang. Applied elasticity. McGraw-Hill Book Company, NY, USA, 1953. 3 